\title{
A Proof Theoretic Approach to Failure in Functional Logic Programming
}

\author{
FRANCISCO J. LÓPEZ-FRAGUAS and JAIME SÁNCHEZ-HERNÁNDEZ * \\ Dep. Sistemas Informáticos y Programación, Univ. Complutense de Madrid \\ E-mail: \{fraguas,jaime\}@sip.ucm.es
}

\begin{abstract}
How to extract negative information from programs is an important issue in logic programming. Here we address the problem for functional logic programs, from a proof-theoretic perspective. The starting point of our work is CRWL (Constructor based ReWriting Logic), a well established theoretical framework for functional logic programming, whose fundamental notion is that of non-strict non-deterministic function. We present a proof calculus, $C R W L F$, which is able to deduce negative information from $C R W L$-programs. In particular, $C R W L F$ is able to prove 'finite' failure of reduction within $C R W L$.
\end{abstract}

$K E Y W O R D S$ : constructive failure, functional logic programming, proof calculi.

Submitted: Dec 22, 2000, revised: Feb 21, 2002, accepted: Sept 272002.

\section{Introduction}

We address in this paper the problem of extracting negative information from functional logic programs. The question of negation is a main topic of research in the logic programming field, and the most common approach is negation as failure (Clark 1978), as an easy effective approximation to the CWA (closed world assumption), which is a simple, but uncomputable, way of deducing negative information from positive programs (see e.g. Apt and Bol (1994) for a survey on negation in logic programming).

On the other hand, functional logic programming ( $F L P$ for short) is a powerful programming paradigm trying to combine the nicest properties of functional and logic programming (see Hanus (1994) for a now 'classical' survey on FLP). A mainstream in current FLP research considers languages which are biased to the functional programming style, in the sense that programs define functions, but having logic programming capabilities because their operational mechanisms are based on narrowing. Some existing systems of this kind are $\mathcal{T O Y}$ (López and Sánchez 1999a Abengózar et al. 2002) or the various implementations of Curry (Hanus 2000). In the rest of the paper we have in mind such approach when we refer to FLP.

FLP subsumes pure logic programming: predicates can be defined as functions

* The authors have been partially supported by the Spanish CICYT (project TIC 2002-01167 'MEHLODIAS'). 
returning the value 'true', for which definite clauses can be written as conditional rewrite rules. In some simple cases it is enough, to handle negation, just to define predicates as two-valued boolean functions returning the values 'true' or 'false'. But negation as failure is far more expressive, as we see in the next section, and it is then of clear interest to investigate a similar notion for the case of FLP. Failure in logic programs, when seen as functional logic programs, corresponds to failure of reduction to 'true'. This generalizes to a natural notion of failure in FLP, which is 'failure of reduction to (partial) data constructor value', or in other terms, 'failure of reduction to head normal form' (hnf for short).

As technical setting for our work we have chosen CRWL (González et al. 1996 González et al. 1999), a well established theoretical framework for FLP. The fundamental notion in $C R W L$ is that of non-strict non-deterministic function, for which $C R W L$ provides a firm logical basis. Instead of equational logic, which is argued to be unsuitable for FLP in González et al. (1999), $C R W L$ considers a Constructor based ReWriting Logic, presented by means of a proof calculus, which determines what statements can be deduced from a given program. In addition to the prooftheoretic semantics, (González et al. 1996 González et al. 1999) develop a model theoretic semantics for $C R W L$, with existence of distinguished free term models for programs, and a sound and complete lazy narrowing calculus as operational semantics. The interest of $C R W L$ as a theoretical framework for FLP has been mentioned in Hanus (2000), and is further evidenced by its many extensions incorporating relevant aspects of declarative programming like HO features (González et al. 1997), polymorphic and algebraic types (Arenas and Rodríguez 2001), or constraints (Arenas et al. 1999). The framework, with many of these extensions (like types, HO and constraints) has been implemented in the system $\mathcal{T O Y}$.

Here we are interested in extending the proof-theoretic side of $C R W L$ to cope with failure. More concretely, we look for a proof calculus, which will be called $C R W L F$ (' $C R W L$ with failure'), which is able to prove failure of reduction in $C R W L$. Since reduction in $C R W L$ is expressed by proving certain statements, our calculus will provide proofs of unprovability within $C R W L$. As for the case of $C W A$, unprovability is not computable, which means that our calculus can only give an approximation, corresponding to cases which can be intuitively described as 'finite failures'.

There are very few works about negation in FLP. In Moreno (1994) the work of Stuckey about constructive negation (Stuckey 1991, Stuckey 1995) is adapted to the case of FLP with strict functions and innermost narrowing as operational mechanism. In Moreno (1996) a similar work is done for the case of non-strict functions and lazy narrowing. The approach is very different of the proof-theoretic view of our work. The fact that we also consider non-deterministic functions makes a significant difference.

The proof-theoretic approach, although not very common, has been followed sometimes in the logic programming field, as in Jäger and Stärk (1998), which develops for logic programs (with negation) a framework which resembles, in a very general sense, $C R W L$ : a program determines a deductive system for which deducibility, validity in a class of models, validity in a distinguished model and derivability by an operational calculus are all equivalent. Our work attempts to be the first step of 
what could be a similar programme for FLP extended with the use of failure when writing programs.

The rest of the paper is organized as follows. In Section 2 we discuss the interest of using failure as a programming construct in the context of FLP. In Section 3 we give the essentials of $C R W L$ which are needed for our work. Section 3 presents the $C R W L F$-calculus, preceded by some illustrative examples. Sections 4,5 and 6 constitute the technical core of the paper, presenting the properties of $C R W L F$ and its relation to $C R W L$. Finally, Section 7 outlines some conclusions and possible future work.

\section{The Interest of Failure in FLP}

Although this work is devoted only to the theoretical aspects of failure in FLP, in this section we argue some possible applications of this resource from the point of view of writing functional logic programs.

$F L P$ combines some of the main capabilities of the two main streams of declarative programming: functional programming $(F P)$ and logic programming $(L P)$. Theoretical aspects of FLP are well established (see e.g. González et al. (1999)) and there are also practical implementations such as Curry or $\mathcal{T O Y}$. Disregarding syntax, both pure Prolog and (a wide subset of) Haskell are subsumed by those systems. The usual claim is then that by the use of an FLP system one can choose the style of programming better suited to each occasion.

However there are features related to failure, mainly in $L P$ (but also in $F P$ ) yet not available in $F L P$ systems. This poses some problems to $F L P$ : if a logic program uses negation (a very common situation), it cannot be seen as an FLP program. This is not a very serious inconvenience if other features of $F L P$ could easily replace the use of failure. But if the FLP solution (without failure) to a problem is significantly more complex than, say, an $L P$ solution making use of failure, then it is not worth to use FLP for that problem, thus contradicting in practice the claim that FLP can successfully replace $L P$ and $F P$.

We now give concrete examples of the potential use of a construction to express failure in FLP programs. We assume for the examples below that we incorporate to FLP the following function to express failure of an expression:

$$
\text { fails }(e)::= \begin{cases}\text { true } & \text { if } e \text { fails to be reduced to hnf } \\ \text { false } & \text { otherwise }\end{cases}
$$

The sensible notion to consider is failure of reduction to head normal form ${ }^{1}$, since head normal forms (i.e., variables or expressions $c(.$.$) , where c$ is a constructor symbol) are the expressions representing, without the need of further reduction, defined (maybe partial) values.

\footnotetext{
1 To be technically more precise, we should speak of 'failure to reduction to head normal form with respect to the $C R W L$-calculus', to be recalled in Section 3.
} 
Example 1 (Failure to express negation in LP)

The most widespread approach to negation in the LP paradigm is negation as failure (Clark 1978), of which all PROLOG systems provide an implementation. Typically, in a logic program one writes clauses defining the positive cases for a predicate, and the effect of using negation is to 'complete' the definition with the negative cases, which correspond to failure of the given clauses.

For example, in $L P$ the predicate member can be defined as:

$$
\begin{aligned}
& \text { member }(X,[X \mid Y s]) . \\
& \text { member }(X,[Y \mid Y s]) \leftarrow \text { member }(X, Y s) .
\end{aligned}
$$

This defines member $(X, L)$ as a semidecision procedure to check if $X$ is an element of $L$. If one needs to check that $X$ is not an element of $L$, then negation can be used, as in the clause

$$
\operatorname{add}(X, L,[X \mid L]):- \text { not member }(X, L) .
$$

Predicates like member can be defined in FLP as true-valued functions, converting clauses into conditional rules returning true:

$$
\begin{aligned}
& \text { member }(X,[Y \mid Y s]) \rightarrow \text { true } \Leftarrow X \bowtie Y \\
& \text { member }(X,[Y \mid Y s]) \rightarrow \text { true } \Leftarrow \text { member }(X, Y s) \bowtie \text { true }
\end{aligned}
$$

To achieve linearity (i.e., no variable repetition) of heads, a usual requirement in FLP, the condition $X \bowtie Y$ is used in the first rule. The symbol $\bowtie$ (taken from (González et al. 1996 González et al. 1999)) is used throughout the paper to express 'joinability', which means that both sides can be reduced to the same data value (for the purpose of this example, $\bowtie$ can be read simply as strict equality).

What cannot be directly translated into FLP (without failure) is a clause like that of add, but with failure it is immediate:

$$
\operatorname{add}\left(X, L,\left[X^{\prime} \mid L^{\prime}\right]\right) \rightarrow \operatorname{true} \Leftarrow \operatorname{fails}(\operatorname{member}(X, L)) \bowtie \operatorname{true}, X^{\prime} \bowtie X, L^{\prime} \bowtie L
$$

In general, any literal of the form not Goal in a logic program can be replaced by fails $($ Goal $) \bowtie$ true in its FLP-translation.

This serves to argue that $F L P$ with failure subsumes $L P$ with negation, but of course this concrete example corresponds to the category of 'dispensable' uses of failure, because there is a natural failure-free FLP counterpart to the predicate member in the form of a bivaluated boolean function, where the failure is expressed by the value false. The following could be such a definition of member:

$$
\begin{array}{ll}
\text { member }(X,[]) & \rightarrow \text { false } \\
\text { member }(X,[Y \mid Y s]) & \rightarrow \text { true } \Leftarrow X \bowtie Y \\
\text { member }(X,[Y \mid Y s]) & \rightarrow \text { member }(X, Y s) \Leftarrow X \diamond Y
\end{array}
$$

The symbol $\diamond$ (corresponding to disequality $\neq$ of (López and Sánchez 1999a López and Sánchez 1999b)) expresses 'divergence', meaning that both sides can be reduced to some extent as to detect inconsistency, i.e., conflict of constructors at the same position (outside function applications). Now add can be easily defined without using failure: 


$$
\operatorname{add}\left(X, L,\left[X^{\prime} \mid L^{\prime}\right]\right) \rightarrow \operatorname{true} \Leftarrow \operatorname{member}(X, L) \bowtie \text { false, } X^{\prime} \bowtie X, L^{\prime} \bowtie L
$$

The next examples show situations where the use of negation is more 'essential', in the sense that it is the natural way (at least a very natural way) of doing things.

Example 2 (Failure in search problems I)

Non-deterministic constructs are a useful way of programming problems involving search. In $F L P$ one can choose to use predicates, as in $L P$, or non-deterministic functions. In these cases, the use of failure can greatly simplify the task of programming. We see an example with non-deterministic functions, a quite specific FLP feature which is known to be useful for programming (Abengózar et al. 2002 Hanus 2000 Antoy 1997) in systems like Curry or $\mathcal{T} \mathcal{O} \mathcal{Y}$.

Consider the problem of deciding, for acyclic directed graphs, if there is a path connecting two nodes. A graph can be represented by a non-deterministic function next, with rules of the form $n \operatorname{ext}(N) \rightarrow N^{\prime}$, indicating that there is an arc from $N$ to $N^{\prime}$. A concrete graph with nodes $a, b, c$ and $d$ could be given by the rules:

$$
\begin{aligned}
& n \operatorname{ext}(a) \rightarrow b \\
& n \operatorname{ext}(a) \rightarrow c \\
& n \operatorname{ext}(b) \rightarrow c \\
& n \operatorname{next}(b) \rightarrow d
\end{aligned}
$$

and to determine if there is a path from $X$ to $Y$ we can define:

$$
\begin{aligned}
& \operatorname{path}(X, Y) \rightarrow \text { true } \Leftarrow X \bowtie Y \\
& \operatorname{path}(X, Y) \rightarrow \operatorname{true} \Leftarrow X \diamond Y, \operatorname{path}(\operatorname{next}(X), Y) \bowtie \text { true }
\end{aligned}
$$

Notice that path behaves as a semidecision procedure recognizing only the positive cases, and there is no clear way (in 'classical' FLP) of completing its definition with the negatives ones, unless we change from the scratch the representation of graphs. Therefore we cannot, for instance, program in a direct way a property like

$$
\text { safe }(X)::=X \text { is not connected with } d
$$

Using failure this is an easy task:

$$
\operatorname{safe}(X) \rightarrow \operatorname{fails}(\operatorname{path}(X, d))
$$

With this definition, safe $(c)$ becomes true, while safe $(a)$, safe $(b)$ and safe $(d)$ are all false.

Example 3 (Failure in search problems II)

We examine now an example mentioned in Apt (2000) as one striking illustration of the power of failure as expressive resource in $L P$. We want to program a twoperson finite game where the players must perform alternate legal moves, until one of them, the loser, cannot move.

We assume that legal moves from a given state are programmed by a nondeterministic function move(State) returning the new state after the movement. Using failure it is easy to program a function to perform a winning movement from a given position, if there is one: 


$$
\begin{aligned}
& \text { winMove }(\text { State }) \rightarrow \text { State }^{\prime} \Leftarrow \text { State }^{\prime} \bowtie \text { move }(\text { State }), \\
& \text { fails }\left(\text { winMove }\left(\text { State }^{\prime}\right)\right) \bowtie \text { true }
\end{aligned}
$$

We think it would be difficult to find a simpler coding without using failure.

As a concrete example we consider the well-known game Nim, where there are some rows of sticks, and each player in his turn must pick up one or more sticks from one of the rows. A player loses when he cannot make a movement, that is, when there are not more sticks because the other player (the winner) has picked up the last one. Nim states can be defined by a list of natural numbers (represented by 0 and $s(-)$ as usual), and the non-deterministic function move can be programmed as:

$$
\begin{aligned}
& \operatorname{move}([N \mid N s]) \rightarrow[\operatorname{pick}(N) \mid N s] \\
& \operatorname{move}([N \mid N s]) \rightarrow[N \mid \operatorname{move}(N s)] \\
& \operatorname{pick}(s(N)) \rightarrow N \\
& \operatorname{pick}(s(N)) \rightarrow \operatorname{pick}(N)
\end{aligned}
$$

A winning move from the state $[s(s(z)), s(z)]$ can be obtained by reducing the expression winMove $([s(s(z)), s(z)])$. The proof calculus presented in Sect. 3.2 can prove that it can be reduced to $[s(z), s(z)]$, and it is easy to check that this move guarantees the victory.

Example 4 (Failure to express default rules)

Compared to the case of $L P$, failure is not a so important programming construct in $F P$. There is still one practical feature of existing $F P$ languages somehow related to failure, which is the possibility of defining functions with default rules. In many $F P$ systems pattern matching determines the applicable rule for a function call, and as rules are tried from top to bottom, default rules are implicit in the definitions. In fact, the $n+1$-th rule in a definition is only applied if the first $n$ rules are not applicable. For example, assume the following definition for the function $f$ :

$$
\begin{array}{ll}
f(0) & \rightarrow 0 \\
f(X) & \rightarrow 1
\end{array}
$$

The evaluation of the expression $f(0)$ in a functional language like Haskell (Peyton-Jones and Hughes 1999), will produce the value 0 by the first rule. The second rule is not used for evaluating $f(0)$, even if pattern matching would succeed if the rule would be considered in isolation. This sequential treatment of rules is useful in some cases, specially for writing 'last' rules covering default cases whose direct formulation with pattern matching could be complicated. But observe that in systems allowing such sequential trials of pattern matching, rules have not a declarative meaning by themselves; their interpretation depends also on the previous rules.

This contrasts with functional logic languages which try to preserve the declarative reading of each rule. In such systems the expression $f(0)$ of the example above is reducible, by applying in a non-deterministic way any of the rules, to the values 0 and 1.

To achieve (and generalize) the effect of default rules in $F L P$, an explicit syntactical construction 'default' can be introduced, as it has been done in (Moreno 1994).

The function $f$ could be defined as: 


$$
\begin{aligned}
& f(0) \rightarrow 0 \\
& \text { default } f(X) \rightarrow 1
\end{aligned}
$$

The intuitive operational meaning is: to reduce a call to $f$ proceed with the first rule for $f$; if the reduction fails then try the default rule.

The problem now is how to achieve this behavior while preserving the equational reading of each rule. Using conditional rewrite rules and our function fails(_), we can transform the definition of a function to eliminate default rules. In the general case we can consider conditional rewrite rules for the original definition. Let $h$ be a function defined as:

$$
\begin{aligned}
& h\left(\bar{t}_{1}\right) \rightarrow e_{1} \Leftarrow \bar{C}_{1} \\
& \ldots \\
& h\left(\bar{t}_{n}\right) \rightarrow e_{n} \Leftarrow \bar{C}_{n} \\
& \text { default } h\left(\bar{t}_{n+1}\right) \rightarrow e_{n+1} \Leftarrow \bar{C}_{n+1}
\end{aligned}
$$

The idea of the transformation is to consider a new function $h^{\prime}$ defined by the first $n$ rules of $h$. The original $h$ will be defined as $h^{\prime}$ if it succeeds and as the default rule if $h^{\prime}$ fails:

$$
\begin{aligned}
& h(\bar{X}) \rightarrow h^{\prime}(\bar{X}) \\
& h(\bar{X}) \rightarrow e_{n+1} \Leftarrow \text { fails }\left(h^{\prime}(\bar{X})\right) \bowtie \text { true, } \bar{C}_{n+1} \\
& h^{\prime}\left(\bar{t}_{1}\right) \rightarrow e_{1} \Leftarrow \bar{C}_{1} \\
& \ldots \\
& h^{\prime}\left(\bar{t}_{n}\right) \rightarrow e_{n} \Leftarrow \bar{C}_{n}
\end{aligned}
$$

Applying this transformation to our function example $f$, we obtain:

$$
\begin{aligned}
& f(X) \rightarrow f^{\prime}(X) \\
& f(X) \rightarrow 1 \Leftarrow \text { fails }\left(f^{\prime}(X)\right) \bowtie \text { true } \\
& f^{\prime}(0) \rightarrow 0
\end{aligned}
$$

With this definition we have got the expected behavior for $f$ without losing the declarative reading of rules.

As another example, we can use a default rule to complete the definition of the function path in the example 2 above:

$$
\begin{aligned}
& \text { path }(X, Y) \rightarrow \text { true } \Leftarrow X \bowtie Y \\
& \text { path }(X, Y) \rightarrow \text { true } \Leftarrow X \diamond Y, \operatorname{path}(\operatorname{next}(X), Y) \bowtie \operatorname{true} \\
& \text { default path }(X, Y) \rightarrow \text { false }
\end{aligned}
$$

The function safe can now be written as:

$$
\operatorname{safe}(X) \rightarrow \operatorname{neg}(\operatorname{path}(X, d))
$$

where neg is the boolean function

$$
\begin{array}{ll}
\text { neg }(\text { true }) & \rightarrow \text { false } \\
\text { neg }(\text { false }) & \rightarrow \text { true }
\end{array}
$$


Notice that in this example the (implicit) condition for applying the default rule of path is far more complex than a merely syntactical default case expressing failure of pattern matching, a feature recently discussed in (Curry mailing list 2000) as useful for FLP. Of course, default rules in the sense of (Moreno 1994) and of this paper also cover such syntactical cases.

\section{The $C R W L$ Framework}

We give here a short summary of (a slight variant of) $C R W L$, in its proof-theoretic face. Model theoretic semantics and lazy narrowing operational semantics are not considered here. Full details can be found in (González et al. 1999, López and Sánchez 1999b).

\subsection{Technical Preliminaries}

We assume a signature $\Sigma=D C_{\Sigma} \cup F S_{\Sigma}$ where $D C_{\Sigma}=\bigcup_{n \in \mathbb{N}} D C_{\Sigma}^{n}$ is a set of constructor symbols and $F S_{\Sigma}=\bigcup_{n \in \mathbb{N}} F S_{\Sigma}^{n}$ is a set of function symbols, all of them with associated arity and such that $D C_{\Sigma} \cap F S_{\Sigma}=\emptyset$. We also assume a countable set $\mathcal{V}$ of variable symbols. We write $\operatorname{Term}_{\Sigma}$ for the set of (total) terms (we say also expressions) built up with $\Sigma$ and $\mathcal{V}$ in the usual way, and we distinguish the subset $C T e r m_{\Sigma}$ of (total) constructor terms or (total) c-terms, which only make use of $D C_{\Sigma}$ and $\mathcal{V}$. The subindex $\Sigma$ will usually be omitted. Terms intend to represent possibly reducible expressions, while c-terms represent data values, not further reducible.

We will need sometimes to use the signature $\Sigma_{\perp}$ which is the result of extending $\Sigma$ with the new constant (0-arity constructor) $\perp$, that plays the role of the undefined value. Over $\Sigma_{\perp}$, we can build up the sets Term $m_{\perp}$ and $C T e r m_{\perp}$ of (partial) terms and (partial) c-terms respectively. Partial c-terms represent the result of partially evaluated expressions; thus, they can be seen as approximations to the value of expressions.

As usual notations we will write $X, Y, Z, \ldots$ for variables, $c, d$ for constructor symbols, $f, g$ for functions, $e$ for terms and $s, t$ for c-terms. In all cases, primes (') and subindices can be used.

We will use the sets of substitutions CSubst $=\{\theta: \mathcal{V} \rightarrow C$ Term $\}$ and $C S u b s t_{\perp}=$ $\left\{\theta: \mathcal{V} \rightarrow C T e r m_{\perp}\right\}$. We write $e \theta$ for the result of applying $\theta$ to $e$.

Given a set of constructor symbols $S$ we say that the c-terms $t$ and $t^{\prime}$ have an $S$-clash if they have different constructor symbols of $S$ at the same position.

\subsection{The Proof Calculus for CRWL}

A $C R W L$-program $\mathcal{P}$ is a finite set of conditional rewrite rules of the form:

$$
\underbrace{f\left(t_{1}, \ldots, t_{n}\right)}_{\text {head }} \rightarrow \underbrace{e}_{\text {body }} \Leftarrow \underbrace{C_{1}, \ldots, C_{m}}_{\text {condition }}
$$

where $f \in F S^{n}$, and fulfilling the following conditions: 
- $\left(t_{1}, \ldots, t_{n}\right)$ is a linear tuple (each variable in it occurs only once) with $t_{1}, \ldots, t_{n} \in$ CTerm;

- $e \in T e r m$;

- each $C_{i}$ is a constraint of the form $e^{\prime} \bowtie e^{\prime \prime}$ (joinability) or $e^{\prime} \diamond e^{\prime \prime}$ (divergence) where $e^{\prime}, e^{\prime \prime} \in$ Term;

- extra variables are not allowed, i.e., all the variables appearing in the body $e$ and the condition $\bar{C}$ must also appear in the head $f(\bar{t})(\operatorname{var}(e) \cup \operatorname{var}(\bar{C}) \subseteq$ $\operatorname{var}(\bar{t}))$. This condition is not required in (González et al. 1996 González et al. 1999); see the end of this section for a discussion of this issue.

The reading of the rule is: $f\left(t_{1}, \ldots, t_{n}\right)$ reduces to $e$ if the conditions $C_{1}, \ldots, C_{n}$ are satisfied. We write $\mathcal{P}_{f}$ for the set of defining rules of $f$ in $\mathcal{P}$.

Given a program $\mathcal{P}$, the proof calculus for $C R W L$ can derive from it three kinds of statements:

- Reduction or approximation statements: $e \rightarrow t$, with $e \in$ Term $_{\perp}$ and $t \in$ $C T e r m_{\perp}$. The intended meaning of such statement is that $e$ can be reduced to $t$, where reduction may be done by applying rewriting rules of $\mathcal{P}$ or by replacing subterms of $e$ by $\perp$. If $e \rightarrow t$ can be derived, $t$ represents one of the possible values of the denotation of $e$.

- Joinability statements: $e \bowtie e^{\prime}$, with $e, e^{\prime} \in T e r m_{\perp}$. The intended meaning in this case is that $e$ and $e^{\prime}$ can be both reduced to some common totally defined value, that is, we can prove $e \rightarrow t$ and $e^{\prime} \rightarrow t$ for some $t \in C T e r m$.

- Divergence statements: $e \diamond e^{\prime}$, with $e, e^{\prime} \in T e r m_{\perp}$. The intended meaning now is that $e$ and $e^{\prime}$ can be reduced to some (possibly partial) c-terms $t$ and $t^{\prime}$ having a $D C$-clash. In (González et al. 1996, González et al. 1999) divergence conditions are not considered. They have been incorporated to CRWL in López and Sánchez (1999b) as a useful and expressive resource for programming that is implemented in the system $\mathcal{T O Y}$.

When using function rules to derive statements, we will need to use what are called $c$-instances of such rules. The set of c-instances of a program rule $R$ is defined as:

$$
[R]_{\perp}=\left\{R \theta \mid \theta \in C \text { Subst }_{\perp}\right\}
$$

Parameter passing in function calls will be expressed by means of these c-instances in the proof calculus.

Table 1 shows the proof calculus for $C R W L$. We write $\mathcal{P} \vdash_{C R W L} \varphi$ for expressing that the statement $\varphi$ is provable from the program $\mathcal{P}$ with respect to this calculus. The rule (4) allows to use c-instances of program rules to prove approximations. These c-instances may contain $\perp$ and by rule (1) any expression can be reduced to $\perp$. This reflects a non-strict semantics. A variable $X$ can only be approximated by itself (rule 2) and by $\perp$ (rule 1), so a variable is similar to a constant in derivations with this calculus. Nevertheless, when using function rules of the program a variable of such rule can take any value by taking the appropriate c-instance. The rule (3) is for term decomposition and rules (5) and (6) corresponds to the definition of $\bowtie$ and $\diamond$ respectively. 
Table 1. Rules for $C R W L$-provability

\begin{tabular}{l}
\hline \hline (1) $\frac{}{e \rightarrow \perp}$ \\
(2) $\frac{}{X \rightarrow X} \quad X \in \mathcal{V}$ \\
(3) $\frac{e_{1} \rightarrow t_{1}, \ldots, e_{n} \rightarrow t_{n}}{c\left(e_{1}, \ldots, e_{n}\right) \rightarrow c\left(t_{1}, \ldots, t_{n}\right)} \quad c \in D C^{n}, \quad t_{i} \in C T e r m_{\perp}$ \\
(4) $\frac{e_{1} \rightarrow s_{1}, \ldots, e_{n} \rightarrow s_{n} \quad C \quad e \rightarrow t}{f\left(e_{1}, \ldots, e_{n}\right) \rightarrow t} \quad$ if $t \neq \equiv \perp, R \in \mathcal{P}_{f}$ \\
(5) $\frac{e \rightarrow t e^{\prime} \rightarrow t}{e \bowtie e^{\prime}} \quad$ if $t \in C T e r m$ \\
(6) $\frac{\left.\left.e \rightarrow t e_{1}, \ldots, s_{n}\right) \rightarrow e \Leftarrow C\right) \in[R]_{\perp}}{e \diamond e^{\prime}} \quad$ if $t, t^{\prime} \in C T e r t_{\perp}$ and have a $D C-$ clash \\
\hline \hline
\end{tabular}

A distinguished feature of $C R W L$ is that functions can be non-deterministic. For example, assuming the constructors $z$ (zero) and $s$ (successor) for natural numbers, a non-deterministic function coin for expressing the possible results of throwing a coin can defined by the rules:

$$
\begin{aligned}
& \text { coin } \rightarrow z \\
& \text { coin } \rightarrow s(z)
\end{aligned}
$$

It is not difficult to see that the previous calculus can derive the statement coin $\rightarrow z$ and also coin $\rightarrow s(z)$. The use of c-instances in rule (4) instead of general instances corresponds to call time choice semantics for non-determinism (see (González et al. 1999)). As an example, in addition to coin consider the functions add and double defined as:

$$
\begin{aligned}
& \operatorname{add}(z, Y) \rightarrow Y \\
& \operatorname{add}(s(X), Y) \rightarrow s(\operatorname{add}(X, Y))
\end{aligned}
$$

It is possible to build a $C R W L$-proof for the statement double(coin) $\rightarrow z$ and also for double (coin $) \rightarrow s(s(z))$, but not for double $($ coin $) \rightarrow s(z)$. As an example of derivation, we show a derivation for double $(\operatorname{coin}) \rightarrow z$; at each step we indicate by a number on the left the rule of the calculus applied:

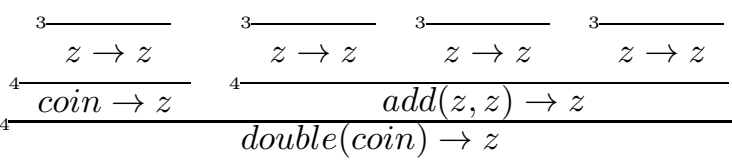

Observe that $\diamond$ is not the logical negation of $\bowtie$. They are not even incompatible: due to non-determinism, two expressions $e, e^{\prime}$ can satisfy both $e \bowtie e^{\prime}$ and $e \diamond e^{\prime}$ 
(although this cannot happen if $e, e^{\prime}$ are c-terms). In the 'coin' example, we can derive both $\operatorname{coin} \bowtie z$ and $\operatorname{coin} \diamond z$.

The denotation of an expression $e$ can be defined as the set of c-terms to which $e$ can be reduced according to this calculus:

$$
\llbracket e \rrbracket=\left\{t \in C T e r m_{\perp} \mid \mathcal{P} \vdash_{C R W L} e \rightarrow t\right\}
$$

For instance, $\llbracket \operatorname{coin} \rrbracket=\{\perp, z, s(\perp), s(z)\}$.

To end our presentation of the $C R W L$ framework we discuss the issue of extra variables (variables not appearing in left hand sides of function rules), which are allowed in (González et al. 1996 González et al. 1999), but not in this paper. This is not as restrictive as it could appear: function nesting can replace the use (typical of logic programming) of variables as repositories of intermediate values, and in many other cases where extra variables represent unknown values to be computed by search, they can be successfully replaced by non-deterministic functions able to compute candidates for such unknown values. A concrete example is given by the function next in example 2 More examples can be found in (González et al. 1999 Abengózar et al. 2002).

The only extra variable we have used in Sect. 2 is $P_{0 s^{\prime}}$ in the definition

$$
\text { winMove }(\text { Pos }) \rightarrow \text { Pos }^{\prime} \Leftarrow \text { Pos }^{\prime} \bowtie \text { move }(\text { Pos }), \text { fails }\left(\text { winMove }\left(\text { Pos }^{\prime}\right)\right) \bowtie \text { true }
$$

of example 3 It can be removed by introducing an auxiliary function:

$$
\begin{aligned}
& \text { winMove }(\text { Pos }) \rightarrow \text { aux }(\text { move }(\text { Pos })) \\
& \text { aux }(\text { Pos }) \rightarrow \text { Pos } \Leftarrow \text { Pos } \bowtie \text { Pos, fails }(\text { winMove }(\text { Pos })) \bowtie \text { true }
\end{aligned}
$$

The effect of the condition Pos $\bowtie$ Pos it to compute a normal form for Pos, which is required in this case to avoid a diverging computation for winMove(Pos).

\section{The $C R W L F$ Framework}

We now address the problem of failure in $C R W L$. Our primary interest is to obtain a calculus able to prove that a given expression fails to be reduced. Since reduction corresponds in $C R W L$ to approximation statements $e \rightarrow t$, we can reformulate our aim more precisely: we look for a calculus able to prove that a given expression $e$ has no possible reduction (other than the trivial $e \rightarrow \perp$ ) in $C R W L$, i.e., $\llbracket e \rrbracket=\{\perp\}$.

Of course, we cannot expect to achieve that with full generality since, in particular, the reason for having $\llbracket e \rrbracket=\{\perp\}$ can be non-termination of the program as rewrite system, a property which is uncomputable. Instead, we look for a suitable computable approximation to the property $\llbracket e \rrbracket=\{\perp\}$, corresponding to cases where failure of reduction is due to 'finite' reasons, which can be constructively detected and managed.

Previous to the formal presentation of the calculus, which will be called $C R W L F$ (for ' $C R W L$ with failure') we give several simple examples for a preliminary understanding of some key aspects of it, and the reasons underlying some of its technicalities. 


\subsection{Some Illustrative Examples}

Consider the following functions, in addition to coin, defined in Sect. 3.2.

$$
f(z) \rightarrow f(z) \quad g(s(s(X))) \rightarrow z \quad \text { h } \rightarrow s(z) \quad h \rightarrow s(h) \quad k(X) \rightarrow z \Leftarrow X \bowtie s(z)
$$

We discuss several situations involving failure with this program:

- The expressions $f(z)$ and $f(s(z))$ fail to be reduced, but for quite different reasons. In the first case $f(z)$ does not terminate. The only possible proof accordingly to $C R W L$ is $f(z) \rightarrow \perp$ (by rule 1 ); any attempt to prove $f(z) \rightarrow t$ with $t \neq \perp$ would produce an 'infinite derivation'. In the second case, the only possible derivation is again $f(s(z)) \rightarrow \perp$, but if we try to prove $f(s(z)) \rightarrow t$ with $t \neq \perp$ we have a kind of 'finite failure': rule 4 needs to solve the parameter passing $s(z) \rightarrow z$, that could be finitely checked as failed, since no rule of the $C R W L$-calculus is applicable. The $C R W L F$-calculus does not prove nontermination of $f(z)$, but will be able to detect and manage the failure for $f(s(z))$. In fact it will be able to perform a constructive proof of this failure.

- Consider now the expression $g(\operatorname{coin})$. Again, the only possible reduction is $g($ coin $) \rightarrow \perp$ and it is intuitively clear that this is another case of finite failure. But this failure is not as simple as in the previous example for $f(s(z))$ : in this case the two possible reductions for coin to defined values are coin $\rightarrow z$ and coin $\rightarrow s(z)$. Both of $z$ and $s(z)$ fail to match the pattern $s(s(X))$ in the rule for $g$, but none of them can be used separately to detect the failure of $g(\operatorname{coin})$. A suitable idea is to collect the set of defined values to which a given expression can be reduced. In the case of coin that set is $\{z, s(z)\}$. The fact that $\mathcal{C}$ is the collected set of values of $e$ is expressed in $C R W L F$ by means of the statement $e \triangleleft \mathcal{C}$. In our example, CRWLF will prove coin $\triangleleft\{z, s(z)\}$. Statements $e \triangleleft \mathcal{C}$ generalize the approximation statements $e \rightarrow t$ of $C R W L$, and in fact can replace them. Thus, CRWLF will not need to use explicit $e \rightarrow t$ statements.

- How far should we go when collecting values? The idea of collecting all values (and to have them completely evaluated) works fine in the previous example, but there are problems when the collection is infinite. For example, according to its definition above, the expression $h$ can be reduced to any positive natural number, so the corresponding set would be $H=\{s(z), s(s(z)), s(s(s(z))), \ldots\}$. Then, what if we try to reduce the expression $f(h)$ ? From an intuitive point of view it is clear that the value $z$ will not appear in $H$, because all its elements have the form $s(\ldots)$. The partial value $\{s(\perp)\}$ is a common approximation to all the elements of $H$. Here we can understand $\perp$ as an incomplete information: we know that all the values for $h$ are successor of 'something', and this implies that they cannot be $z$, which suffices for proving the failure of $f(h)$. The $C R W L F$-calculus will be able to prove the statement $h \triangleleft\{s(\perp)\}$, and we say that $\{s(\perp)\}$ is a Sufficient Approximation Set $(S A S)$ for $h$.

In general, an expression will have multiple $S A S$ 's. Any expression has $\{\perp\}$ as its simplest $S A S$. And, for example, the expression $h$ has an infinite number 
of $S A S^{\prime}$ s: $\{\perp\},\{s(\perp)\},\{s(z), s(s(\perp))\}, \ldots$ The $S A S$ 's obtained by the calculus for coin are $\{\perp\},\{\perp, s(\perp)\},\{\perp, s(z)\},\{z, \perp\},\{z, s(\perp)\}$ and $\{z, s(z)\}$. The $C R W L F$-calculus provides appropriate rules for working with $S A S$ 's. The derivation steps will be guided by these $S A S$ 's in the same sense that $C R W L$ is guided by approximation statements.

- Failure of reduction is due in many cases to failure in proving the conditions in the program rules. The calculus must be able to prove those failures. Consider for instance the expression $k(z)$. In this case we would try to use the c-instance $k(z) \rightarrow z \Leftarrow z \bowtie s(z)$ that allows to perform parameter passing. But the condition $z \bowtie s(z)$ is clearly not provable, so $k(z)$ must fail. For achieving it we must be able to give a proof for ' $z \bowtie s(z)$ cannot be proved with respect to $C R W L$ '. For this purpose we introduce a new constraint $e \bowtie e^{\prime}$ that will be true if we can build a proof of non-provability for $e \bowtie e^{\prime}$. In our case, $z \bowtie s(z)$ is clear because of the clash of constructors. In general the proof for a constraint $e \bowtie e^{\prime}$ will be guided by the corresponding $S A S$ 's for $e$ and $e^{\prime}$ as we will see in the next section. As our initial $C R W L$ framework also allows constraints of the form $e \diamond e^{\prime}$, we need also another constraint $\phi$ for expressing 'failure of $\diamond$ '.

- There is another important question to justify: we use an explicit representation for failure by means of the new constant symbol F. Let us examine some examples involving failures. First, consider the expression $g(s(f(s(z))))$; for reducing it we would need to do parameter passing, i.e., matching $s(f(s(z)))$ with some c-instance of the pattern $s(s(X))$ of the definition of $g$. As $f(s(z))$ fails to be reduced the parameter passing must also fail. If we take $\{\perp\}$ as an $S A S$ for $f(s(z))$ we have not enough information for detecting the failure (nothing can be said about the matching of $s(s(X))$ and $s(\perp)$ ). But if we take $\{\mathrm{F}\}$ as an $S A S$ for $f(s(z))$, this provides enough information to ensure that $s(\mathrm{~F})$ cannot match any c-instance of the pattern $s(s(X))$. Notice that we allow the value $F$ to appear inside the term $s(F)$. One could think that the information $s(\mathrm{~F})$ is essentially the same of $\mathrm{F}$ (for instance, $\mathrm{F}$ also fails to match any c-instance of $s(s(X)))$, but this is not true in general. For instance, the expression $g(s(s(f(s(z)))))$ is reducible to $z$. But if we take the $S A S\{\mathrm{~F}\}$ for $f(s(z))$ and we identify the expression $s(s(f(s(z))))$ with $\mathrm{F}$, matching with the rule for $g$ would not succeed, and the reduction of $g(s(s(f(s(z)))))$ would fail.

We can now proceed with the formal presentation of the $C R W L F$-calculus.

\subsection{Technical Preliminaries}

For dealing with failure we consider two new syntactical elements in CRWLF: a function fails and a constant $\mathrm{F}$. The first one is directly included into the signature, so we consider $\Sigma=D C \cup F S \cup\{$ fails $\}$, where $D C$ and $F S$ are sets of constructor symbols and (user-defined) functions respectively. This symbol, fails, stands for a predefined function whose intuitive meaning is: 


$$
\text { fails }(e)::= \begin{cases}\text { true } & \text { if } e \text { fails to be reduced to hnf } \\ \text { false } & \text { otherwise }\end{cases}
$$

The boolean constants true and false must belong to $D C$, as they are needed to define the function fails. The formal interpretation of this function will be defined by specific rules at the level of the proof-calculus (Table 2).

The second syntactical element, the constant $F$, is introduced as an extension of the signature (as it was the element $\perp$ in $C R W L$ ). So we use the extended signature $\Sigma_{\perp, F}=\Sigma \cup\{\perp, F\}$. We do not include it directly in the signature $\Sigma$ because its role is to express failure of reduction and it is not allowed to appear explicitly in a program. In the case of the function fails we want to allow to use it in programs as we have seen in the examples of Sect. 2

The sets $\operatorname{Term}_{\perp, \mathrm{F}}, C T e r m_{\perp, \mathrm{F}}$ are defined in the natural way, and also the set of substitutions $C$ Subst $_{\perp, \mathrm{F}}=\left\{\theta: \mathcal{V} \rightarrow C\right.$ Term $\left._{\perp, \mathrm{F}}\right\}$.

A natural approximation ordering $\sqsubseteq$ over Term $_{\perp, \mathrm{F}}$ can be defined as the least partial ordering over Term $_{\perp, \mathrm{F}}$ satisfying the following properties:

- $\perp \sqsubseteq e$ for all $e \in$ Term $_{\perp, \mathrm{F}}$,

- $h\left(e_{1}, \ldots, e_{n}\right) \sqsubseteq h\left(e_{1}^{\prime}, \ldots, e_{n}^{\prime}\right)$, if $e_{i} \sqsubseteq e_{i}^{\prime}$ for all $i \in\{1, \ldots, n\}, h \in D C \cup F S \cup\{$ fails $\}$

The intended meaning of $e \sqsubseteq e^{\prime}$ is that $e$ is less defined or has less information than $e^{\prime}$. Two expressions $e, e^{\prime} \in$ Term $_{\perp, \mathrm{F}}$ are consistent if they can be refined to obtain the same information, i.e., if there exists $e^{\prime \prime} \in$ Term $_{\perp, \mathrm{F}}$ such that $e \sqsubseteq e^{\prime \prime}$ and $e^{\prime} \sqsubseteq e^{\prime \prime}$.

Notice that the only relations satisfied by $\mathrm{F}$ are $\perp \sqsubseteq \mathrm{F}$ and $\mathrm{F} \sqsubseteq \mathrm{F}$. In particular, $\mathrm{F}$ is maximal. This is reasonable, since $F$ represents 'failure of reduction' and this gives no further refinable information about the result of the evaluation of an expression. This contrasts with the status given to failure in (Moreno 1996), where $F$ is chosen to verify $\mathrm{F} \sqsubseteq t$ for any $t$ different from $\perp$.

We will frequently use the following notation: given $e \in T e r m_{\perp, \mathrm{F}}, \hat{e}$ stands for the result of replacing by $\perp$ all the occurrences of $\mathrm{F}$ in $e$ (notice that $\hat{e} \in$ Term $_{\perp}$, and $e=\hat{e}$ iff $e \in$ Term $_{\perp}$ ).

\subsection{The Proof Calculus for CRWLF}

Programs in $C R W L F$ are sets of rules with the same form as in $C R W L$, but now they can make use of the function fails in the body and in the condition part, i.e., $C R W L F$ extends the class of programs of $C R W L$ by allowing the use of fails in programs. On the other hand, in $C R W L F$ five kinds of statements can be deduced:

- $e \triangleleft \mathcal{C}$, intended to mean ' $\mathcal{C}$ is an $S A S$ for $e$ '.

- $e \bowtie e^{\prime}, e \diamond e^{\prime}$, with the same intended meaning as in $C R W L$.

- $e \bowtie e^{\prime}, e \phi e^{\prime}$, intended to mean failure of $e \bowtie e^{\prime}$ and $e \diamond e^{\prime}$ respectively.

We will sometimes speak of $\bowtie, \diamond, \bowtie, \phi$ as 'constraints', and use the symbol $\diamond$ to refer to any of them. The constraints $\bowtie$ and $\bowtie$ are called the complementary of each other; the same holds for $\phi$ and $\diamond$, and we write $\widetilde{\diamond}$ for the complementary of $\diamond$. 
When proving a constraint $e \diamond e^{\prime}$ the calculus $C R W L F$ will evaluate an $S A S$ for the expressions $e$ and $e^{\prime}$. These $S A S^{\prime}$ 's will consist of c-terms from $C T e r m_{\perp, \mathrm{F}}$, and provability of the constraint $e \diamond e^{\prime}$ depends on certain syntactic (hence decidable) relations between those c-terms. Actually, the constraints $\bowtie, \diamond, \bowtie$ and $\phi$ can be seen as the result of generalizing to expressions the relations $\downarrow, \uparrow, \downarrow$ and $\forall$ on c-terms, which we define now.

Definition 1 (Relations over CTerm ${ }_{\perp, \mathrm{F}}$ )

- $t \downarrow t^{\prime} \Leftrightarrow_{\text {def }} t=t^{\prime}, t \in C T e r m$

- $t \uparrow t^{\prime} \Leftrightarrow_{\text {def }} t$ and $t^{\prime}$ have a $D C$-clash

- $t \Downarrow t^{\prime} \Leftrightarrow_{\text {def }} t$ or $t^{\prime}$ contain $\mathrm{F}$ as subterm, or they have a $D C$-clash

- $\forall$ is defined as the least symmetric relation over $C T$ Term $m_{\perp, \mathrm{F}}$ satisfying:

i) $X \forall X$, for all $X \in \mathcal{V}$

ii) $\mathrm{F} \forall t$, for all $t \in C T e r m_{\perp, \mathrm{F}}$

iii) if $t_{1} \ngtr t_{1}^{\prime}, \ldots, t_{n} \ngtr t_{n}^{\prime}$ then $c\left(t_{1}, \ldots, t_{n}\right) \ngtr y\left(t_{1}^{\prime}, \ldots, t_{n}^{\prime}\right)$, for $c \in D C^{n}$

The relations $\downarrow$ and $\uparrow$ do not take into account the presence of F, which behaves in this case as $\perp$. The relation $\downarrow$ is strict equality, i.e., equality restricted to total c-terms. It is the notion of equality used in lazy functional or functional-logic languages as the suitable approximation to 'true' equality $(=)$ over $C T e r m_{\perp}$. The relation $\uparrow$ is a suitable approximation to ' $\neg=$ ', and hence to ' $\neg \downarrow$ ' (where $\neg$ stands for logical negation). The relation $\downarrow$ is also an approximation to ' $\neg \downarrow$ ', but in this case using failure information ( $\downarrow$ can be read as ' $\downarrow$ fails'). Notice that $\downarrow$ does not imply ' $\neg=$ ' anymore (we have, for instance, $F \downarrow$ F). Similarly, $\not$ is also an approximation to ' $\neg \uparrow$ ' which can be read as ' $\uparrow$ fails'.

The following proposition reflects these and more good properties of $\downarrow, \uparrow, \downarrow, \not$.

\section{Proposition 1}

The relations $\downarrow, \uparrow, \downarrow, \nsucc$ satisfy

a) For all $t, t^{\prime}, s, s^{\prime} \in C T e r m_{\perp, \mathrm{F}}$

i) $t \downarrow t^{\prime} \Leftrightarrow \hat{t} \downarrow \hat{t}^{\prime}$ and $t \uparrow t^{\prime} \Leftrightarrow \hat{t} \uparrow \hat{t}^{\prime}$

ii) $t \uparrow t^{\prime} \Rightarrow t \downarrow t^{\prime} \Rightarrow \neg\left(t \downarrow t^{\prime}\right)$

iii) $t \downarrow t^{\prime} \Rightarrow t \ngtr t^{\prime} \Rightarrow \neg\left(t \uparrow t^{\prime}\right)$

b) $\downarrow, \uparrow, \downarrow, \forall$ are monotonic, i.e., if $t \sqsubseteq s$ and $t^{\prime} \sqsubseteq s^{\prime}$ then: $t \Re t^{\prime} \Rightarrow s \Re s^{\prime}$, where $\Re \in$ $\{\downarrow, \uparrow, \downarrow, \downarrow\}$. Furthermore $\bigvee_{G}$ and $\bigvee_{G}$ are the greatest monotonic approximations to $\neg \downarrow_{G}$ and $\neg \uparrow_{G}$ respectively, where $\Re_{G}$ is the restriction of $\Re$ to the set of ground (i.e., without variables) c-terms from $C T e r m_{\perp, \mathrm{F}}$.

c) $\downarrow$ and $\bigvee$ are closed under substitutions from CSubst; $\downarrow$ and $\uparrow$ are closed under substitutions from CSubst $_{\perp, \mathrm{F}}$ 
Proof

We prove each property separately:

a)

i) $-t \downarrow t^{\prime} \Leftrightarrow \hat{t} \downarrow \hat{t}^{\prime}$ : two terms satisfying the relation $\downarrow$ cannot contain $\perp$ neither $\mathrm{F}$. Hence $t=\hat{t}$ and $t^{\prime}=\hat{t}^{\prime}$, and the equivalence is trivial.

- $t \uparrow t^{\prime} \Leftrightarrow \hat{t} \uparrow \hat{t}^{\prime}$ : the relation $\uparrow$ is satisfied when the terms have a $D C$-clash at some position $p$; since $t$ and $\hat{t}\left(t^{\prime}\right.$ and $\hat{t}^{\prime}$ resp.) have the same constructor symbols at the same positions, the equivalence is clear.

ii) The implication $t \uparrow t^{\prime} \Rightarrow t \downarrow t^{\prime}$ is clear from definitions of $\uparrow$ and $\downarrow$. For $t \downarrow t^{\prime} \Rightarrow \neg\left(t \downarrow t^{\prime}\right)$ : if $t \Downarrow t^{\prime}$ then either $\mathrm{F}$ appears in $t$ or $t^{\prime}$, or $t$ and $t^{\prime}$ have a $D C$-clash. In both cases $t \downarrow t^{\prime}$ does not hold.

iii) For $t \downarrow t^{\prime} \Rightarrow t \bigvee t^{\prime}$ : if $t \downarrow t^{\prime}$ then $t=t^{\prime}$ with $t \in C$ Term and we have $t \bigvee t^{\prime}$ by applying repeatedly $i$ ) and iii) of the definition of $\not$. For $t \forall t^{\prime} \Rightarrow \neg\left(t \uparrow t^{\prime}\right)$ let us assume $t \bigvee t^{\prime}$ and proceed by induction on the depth $d$ of $t$ :

$\underline{d=0}$ : if $t=\perp$ or $t=\mathrm{F}$ then $t$ and $t^{\prime}$ cannot have any $D C$-clash and then $t \uparrow t^{\prime}$ is not true. If $t=X$ or $t=c \in D C^{0}$ then $t \gamma t^{\prime}$ implies that $t^{\prime}=\mathrm{F}$ or $t^{\prime}=t$; therefore $t$ and $t^{\prime}$ cannot have any $D C$-clash and $t \uparrow t^{\prime}$ is not true.

$\underline{d \Rightarrow d+1}$ : if $t=c\left(t_{1}, \ldots, t_{n}\right)$, then either $t^{\prime}=\mathrm{F}$ and $t \uparrow t^{\prime}$ is not true, or $t^{\prime}=c\left(t_{1}^{\prime}, \ldots, t_{n}^{\prime}\right)$ with $t_{i} \ngtr t_{i}^{\prime}$ for all $i \in\{1, \ldots, n\}$; in this case, by i.h. there is not a pair $\left(t_{i}, t_{i}^{\prime}\right)$ with a $D C$-clash, so neither $t$ and $t^{\prime}$ have $D C$-clashes, and therefore $t \uparrow t^{\prime}$ is not true.

b) We prove monotonicity for each relation:

- For $\downarrow$ : by definition of $\downarrow$, if $t \downarrow t^{\prime}$ then $t, t^{\prime} \in C T e r m$ (they are maximal with respect to $\sqsubseteq)$, hence $s=t$ and $s^{\prime}=t^{\prime}$ and then $s \downarrow s^{\prime}$.

- For $\uparrow$ : if $t \uparrow t^{\prime}$ then $t$ and $t^{\prime}$ have a $D C$-clash at some position. As $t \sqsubseteq s$ and $t^{\prime} \sqsubseteq s^{\prime}$, then $s$ and $s^{\prime}$ will have the same $D C$-clash at the same position, so $s \uparrow s^{\prime}$.

- For $\downarrow:$ if $t$ and $t^{\prime}$ have a $D C$-clash, $s$ and $s^{\prime}$ will contain the same $D C$-clash, as in $i$ ). If one of them has $\mathrm{F}$ as subterm, by definition of $\sqsubseteq$ it is clear that $s$ or $s^{\prime}$ will also contain $\mathrm{F}$, so $s \Downarrow s^{\prime}$.

- For $\uparrow:$ Here we proceed by induction on the depth $d$ of the term $t$ :

$\underline{d=0}$ : let us check the possibilities for $t$. If $t=X$ or $t=c \in D C^{0}$, then $t \forall t^{\prime}$ implies $t^{\prime}=t$ or $t^{\prime}=\mathrm{F}$; since $t, t^{\prime}$ are maximal with respect to $\sqsubseteq$, then $s=t$ and $s^{\prime}=t^{\prime}$, so we will also have $s \ngtr s^{\prime}$. If $t=\mathrm{F}$ then $s=\mathrm{F}$ and then it is clear that $s \ngtr s^{\prime}$. If $t=\perp$ then $t^{\prime}=\mathrm{F}=s^{\prime}$ and it is clear that $s \not s^{\prime}$.

$d \Rightarrow d+1$ : in this case $t=c\left(t_{1}, \ldots, t_{n}\right)$ and then either $t^{\prime}=\mathrm{F}$, what implies $\overline{s^{\prime}=\mathrm{F} \text { and }}$ then $s \ngtr s^{\prime}$, or $t^{\prime}=c\left(t_{1}^{\prime}, \ldots, t_{n}^{\prime}\right)$ with $t_{i} \forall t_{i}^{\prime}$ for all $i \in\{1, \ldots, n\}$. From $t \sqsubseteq s$ and $t^{\prime} \sqsubseteq s^{\prime}$ it follows that $s=c\left(s_{1}, \ldots, s_{n}\right)$ and $s^{\prime}=c\left(s_{1}^{\prime}, \ldots, s_{n}^{\prime}\right)$, and by i.h. we have $s_{i} \bigvee s_{i}^{\prime}$ for all $i \in\{1, \ldots, n\}$, what implies $s \not y s^{\prime}$.

Now we prove that $\downarrow_{G}$ and $\bigvee_{G}$ are the greatest monotonic approximations to $\neg \downarrow_{G}$ and $\neg \uparrow_{G}$ respectively. We note by $G C T e r m_{\perp, \mathrm{F}}$ the set of all ground $t \in C T e r m_{\perp, \mathrm{F}}$.

- For $\downarrow_{G}$, assume that a relation $R \subseteq\left(G C T e r m_{\perp, \mathrm{F}} \times G C T e r m_{\perp, \mathrm{F}}\right)$ verifies

$$
\begin{aligned}
& t R t^{\prime} \Rightarrow \neg\left(t \downarrow_{G} t^{\prime}\right) \\
& t \sqsubset s, t^{\prime} \sqsubseteq s^{\prime}, t R t^{\prime} \Rightarrow s R s^{\prime}
\end{aligned}
$$


We must prove that $R$ is included in $\Downarrow_{G}$, that is: $\left(t R t^{\prime} \Rightarrow t \downarrow_{G} t^{\prime}\right)$, for any $t, t^{\prime} \in G C T e r m_{\perp, \mathrm{F}}$. We reason by contradiction. Assume $t R t^{\prime}$ and $\neg\left(t \downarrow_{G} t^{\prime}\right)$. Then, by definition of $\downarrow_{G}, t$ and $t^{\prime}$ do not contain $\mathrm{F}$ and do not have a $D C$-clash. Then either $t=t^{\prime}$, or $t$ and $t^{\prime}$ differ because at some positions one of them has $\perp$ while the other has not. In both cases it is easy to see that there exists $s \in G C T e r m$ (totally defined) such that $t \sqsubseteq s$ and $t^{\prime} \sqsubseteq s$. By monotonicity of $R$ we have $s R s$ what implies $\neg\left(s \downarrow_{G} s\right)$, what is a contradiction, since $s \in C T$ erm.

- For $\bigvee_{G}$ we proceed in a similar way as in the previous point: assuming that $R \subseteq\left(G C T e r m_{\perp, \mathrm{F}} \times G C T e r m_{\perp, \mathrm{F}}\right)$ verifies

$$
\begin{aligned}
& t R t^{\prime} \Rightarrow \neg\left(t \uparrow_{G} t^{\prime}\right) \\
& t \sqsubset s, t^{\prime} \sqsubseteq s^{\prime}, t R t^{\prime} \Rightarrow s R s^{\prime}
\end{aligned}
$$

we must prove $\left(t R t^{\prime} \Rightarrow t \bigvee_{G} t^{\prime}\right)$. But if $t R t^{\prime}$ then $\neg\left(t \uparrow_{G} t^{\prime}\right)$, so $t$ and $t^{\prime}$ cannot have any $D C$-clash. They could contain F as subterm but then, by $i i)$ and $i i i)$ of the definition of $\forall$, we will have $t \forall_{G} t^{\prime}$.

c) The property is clear for $\downarrow$ : if we replace in a c-term all the occurrences of a variable by a totally defined c-term, we will obtain a totally defined c-term. For $\uparrow$, such substitution preserves the $D C$-clash of the original c-terms.

For $\downarrow$, if some of the original c-terms had $\mathrm{F}$ as a subterm, the substitution preserves this occurrence of F. On the other hand, if they had a $D C$-clash, then it is clear that this clash will also be present under the substitution.

For $\not$, suppose $t \bigvee t^{\prime}$ and $\theta \in C S u s b t_{\perp, F}$; we proceed by induction on the depth $d$ of the term $t$ :

$\underline{d=0}$ : if $t=\mathrm{F}$, then $t \theta=\mathrm{F}$ and it is clear that $t \theta \forall t^{\prime} \theta$. For the cases $t=X$ and $t=c \in D C^{0}$ we have two possibilities for $t^{\prime}: t^{\prime}=\mathrm{F}$ or $t^{\prime}=t$; if $t^{\prime}=\mathrm{F}$ the result is clear. If we have $t=t^{\prime}=X$ it is not difficult to prove that $X \theta \forall X \theta$ by applying repeatedly $i$ ) and $i$ iii) of definition of $\mathcal{Y}$. The last case, if $t=t^{\prime}=c \in D C^{0}$ is trivial because $\theta$ does not change the terms.

$d \Rightarrow d+1$ : in this case $t=c\left(t_{1}, \ldots, t_{n}\right)$. If $t^{\prime}=\mathrm{F}$ the proof is as in the base case, otherwise $t^{\prime}=c\left(t_{1}^{\prime}, \ldots, t_{n}^{\prime}\right)$ with $t_{i} \bigvee t_{i}^{\prime}$ for all $i \in\{1, \ldots, n\}$. By i.h. we have $t_{i} \theta \forall t_{i}^{\prime} \theta$ and then, by iii) of the definition of $\gamma$ we will have $t \theta \gamma t^{\prime} \theta$.

By (b), we can say that $\downarrow, \uparrow, \downarrow, \ngtr$ behave well with respect to the information ordering: if they are true for some terms, they remain true if we refine the information contained in the terms. Furthermore, (b) states that $\downarrow, \forall$ are defined 'in the best way' (at least for ground c-terms) as computable approximations to $\neg \downarrow$ and $\neg \uparrow$. For c-terms with variables, we must take care: for instance, given the constructor $z$, we have $\neg(X \downarrow z)$, but not $X \Downarrow z$. Actually, to have $X \Downarrow z$ would violate a basic intuition about free variables in logical statements: if the statement is true, it should be true for any value (taken from an appropriate range) substituted for its free variables. The part (c) shows that the definitions of $\downarrow, \uparrow, \downarrow, \downarrow$ respect such principle. Propositions 2 and 3 of the next section show that monotonicity and closure by substitutions are preserved when generalizing $\downarrow, \uparrow, \downarrow, \downarrow$ to $\bowtie, \diamond, \bowtie, \varnothing$. 
We can present now the proof rules for the $C R W L F$-calculus, which are shown in Table 2 The rules 6 and 7 use a generalized notion of c-instances of a rule $R$ : $[R]_{\perp, \mathrm{F}}=\left\{R \theta \mid \theta \in C S u b s t_{\perp, \mathrm{F}}\right\}$. We will use the notation $\mathcal{P} \vdash_{C R W L F} \varphi\left(\mathcal{P} \nvdash_{C R W L F} \varphi\right.$ resp.) for expressing that the statement $\varphi$ is provable (is not provable resp.) with respect to the calculus $C R W L F$ and the program $\mathcal{P}$. CRWLF-derivations have a tree structure (see e.g. Example 5); many results in the following sections use induction over the size of the derivation, i.e., the number of nodes in the derivation tree, which corresponds to the number of inference steps.

The first three rules are analogous to those of the $C R W L$-calculus, now dealing with $S A S$ 's instead of simple approximations (notice the cross product of $S A S$ 's in rule 3 ). Rule 4 is a complex rule which requires some explanation to make clear its reading and, more importantly, its decidability: to obtain an $S A S \mathcal{C}$ for an expression $f\left(e_{1}, \ldots, e_{n}\right)$ (that is, to derive $\left.f\left(e_{1}, \ldots, e_{n}\right) \triangleleft \mathcal{C}\right)$ we must first obtain $S A S^{\prime}$ 's for $e_{1}, \ldots, e_{n}$ (that is, we must derive $e_{1} \triangleleft \mathcal{C}_{1}, \ldots, e_{n} \triangleleft \mathcal{C}_{n}$ ); then for each combination $\bar{t}$ of values in these $S A S$ 's (that is, for each $\bar{t} \in \mathcal{C}_{1} \times \ldots \times \mathcal{C}_{n}$ ) and each program rule $R$ for $f$, a part $\mathcal{C}_{R, \bar{t}}$ of the whole $S A S$ is produced; the union of all these partial $S A S$ 's constitutes the final $S A S \mathcal{C}$ for $f(\bar{e})$. Notice that since $S A S$ 's are finite sets and programs are finite sets of rules, then there is a finite number of $\mathcal{C}_{R, \bar{t}}$ to be calculated in the premises of the rule, and the union of all of them (the final calculated $S A S$ in the rule) is again a finite set ${ }^{2}$.

Rule 4 is quite different from rule 4 in $C R W L$, where we could use any c-instance of any rule for $f$; here we need to consider simultaneously the contribution of each rule to achieve 'complete' information about the values to which the expression can be evaluated. We use the notation $f(\bar{t}) \triangleleft_{R} \mathcal{C}$ to indicate that only the rule $R$ is used to produce $\mathcal{C}$.

Rules 5 to 8 consider all the possible ways in which a concrete rule $R$ can contribute to the $S A S$ of a call $f(\bar{t})$, where the arguments $\bar{t}$ are all in $C T e r m_{\perp, \mathrm{F}}$ (they come from the evaluation of the arguments of a previous call $f(\bar{e})$ ). Rules 5 and 6 can be viewed as positive contributions. The first one obtains the trivial $S A S$ and 6 works if there is a c-instance of the rule $R$ with a head identical to the head of the call (parameter passing); in this case, if the constraints of this c-instance are provable, then the resulting $S A S$ is generated by the body of the c-instance. Rules 7 and 8 consider the negative or failed contributions. Rule 7 applies when parameter passing can be done, but it is possible to prove the complementary $e_{i} \tilde{\diamond} e_{i}^{\prime}$ of one of the constraints $e_{i} \diamond e_{i}^{\prime}$ in the condition of the used c-instance. In this case the constraint $e_{i} \diamond e_{i}^{\prime}$ (hence the whole condition in the c-instance) fails. Finally, rule 8 considers the case in which parameter passing fails because of a $D C \cup\{\mathrm{F}\}$-clash between one of the arguments in the call and the corresponding pattern in $R$.

We remark that for given $f(\bar{t})$ and $R$, the rule 5 and at most one of rules 6 to 8 are applicable. This fact, although intuitive, is far from being trivial to prove and constitutes in fact an important technical detail in the proofs of the results in the next section.

2 To be more precise, this reasoning would be the essential part of an inductive proof of finiteness of $S A S$ 's. But we do not think necessary to burden the reader with such formality. 
Table 2. Rules for $C R W L F$-provability

\begin{tabular}{|c|c|}
\hline (1) & $e \triangleleft\{\perp\}$ \\
\hline (2) & $\frac{X \triangleleft\{X\}}{X \in \mathcal{V}}$ \\
\hline (3) & $\frac{e_{1} \triangleleft \mathcal{C}_{1} \quad \ldots \quad e_{n} \triangleleft \mathcal{C}_{n}}{c\left(e_{1}, \ldots, e_{n}\right) \triangleleft\left\{c\left(t_{1}, \ldots, t_{n}\right) \mid \bar{t} \in \mathcal{C}_{1} \times \ldots \times \mathcal{C}_{n}\right\}} \quad c \in D C^{n} \cup\{\mathrm{F}\}$ \\
\hline (4) & $\frac{e_{1} \triangleleft \mathcal{C}_{1} \quad \ldots \quad e_{n} \triangleleft \mathcal{C}_{n} \quad \ldots \quad f(\bar{t}) \triangleleft_{R} \mathcal{C}_{R, \bar{t}} \quad \ldots}{f\left(e_{1}, \ldots, e_{n}\right) \triangleleft \bigcup_{R \in \mathcal{P}_{f}, \bar{t} \in \mathcal{C}_{1} \times \ldots \times \mathcal{C}_{n}} \mathcal{C}_{R, \bar{t}}} \quad f \in F S^{n}$ \\
\hline (5) & $f(\bar{t}) \triangleleft_{R}\{\perp\}$ \\
\hline (6) & $\frac{e \triangleleft \mathcal{C} \quad \bar{C}}{f(\bar{t}) \triangleleft_{R} \mathcal{C}} \quad(f(\bar{t}) \rightarrow e \Leftarrow \bar{C}) \in[R]_{\perp, \mathrm{F}}$ \\
\hline (7) & $\left(f(\bar{t}) \rightarrow e \Leftarrow \ldots, e_{i} \diamond e_{i}^{\prime}, \ldots\right) \in[R]_{\perp, \mathrm{F}}$, where $i \in\{1, \ldots, n\}$ \\
\hline (8) & $\begin{array}{ll} & R \equiv\left(f\left(s_{1}, \ldots, s_{n}\right) \rightarrow e \Leftarrow \bar{C}\right), t_{i} \text { and } s_{i} \text { have a } \\
f\left(t_{1}, \ldots, t_{n}\right) \triangleleft_{R}\{\mathrm{~F}\} & D C \cup\{\mathrm{F}\} \text {-clash for some } i \in\{1, \ldots, n\}\end{array}$ \\
\hline (9) & $\frac{e \triangleleft \mathcal{C} \quad e^{\prime} \triangleleft \mathcal{C}^{\prime}}{e \bowtie e^{\prime}}$ \\
\hline (10) & $\frac{e \triangleleft \mathcal{C} \quad e^{\prime} \triangleleft \mathcal{C}^{\prime}}{e \diamond e^{\prime}}$ \\
\hline (11) & $\frac{e \triangleleft \mathcal{C} \quad e^{\prime} \triangleleft \mathcal{C}^{\prime}}{e \bowtie e^{\prime}}$ \\
\hline (12) & $\frac{e \triangleleft \mathcal{C} \quad e^{\prime} \triangleleft \mathcal{C}^{\prime}}{e \phi e^{\prime}}$ \\
\hline (13) & $\frac{e \triangleleft\{\mathrm{F}\}}{\text { fails }(e) \triangleleft\{\text { true }\}}$ \\
\hline (14) & $\frac{e \triangleleft \mathcal{C}}{\text { fails }(e) \triangleleft\{\text { false }\}}$ \\
\hline
\end{tabular}

Rules 9 to 12 deal with constraints. With the use of the relations $\downarrow, \uparrow, \downarrow, \forall$ introduced in Sect. 3.3 the rules are easy to formulate. For $e \bowtie e^{\prime}$ it is sufficient to find two c-terms in the $S A S$ 's verifying the relation $\downarrow$, what in fact is equivalent to find a common totally defined c-term such that both expressions $e$ and $e^{\prime}$ can be reduced 
to it (observe the analogy with rule 5 of $C R W L$ ). For the complementary constraint $\downarrow$ we need to use all the information of $S A S$ 's in order to check the relation $\downarrow$ over all the possible pairs. The explanation of rules 10 and 12 is quite similar.

Finally rules 13 and 14 provide together a formal definition of the function fails supported by the notion of $S A S$. Notice that the $S A S$ 's $\{\perp\}$ or $\{\perp, F\}$ do not provide enough information for reducing a call to fails. The call fails $(e)$ is only reduced to $\{$ true $\}$ when every possible reduction of the expression $e$ is failed; and it is reduced to $\{$ false $\}$ there is some reduction of $e$ to some (possible partial) c-term of the form $c(\ldots)(c \in D C)$ or $X$.

The next example shows a derivation of failure using the $C R W L F$-calculus.

\section{Example 5}

Let us consider a program $\mathcal{P}$ with the constructors $z, s$ for natural numbers, [ ] and ':' for lists (although we use Prolog-like notation for them, that is, $[z, s(z) \mid L]$ represents the list $(z:(s(z): L)))$ and also the constructors $\mathrm{t}$, $\mathrm{f}$ that represent the boolean values true and false. Assume the functions coin and $h$ defined in Sect. B.2 and Sect. 4.1 respectively and also the function $m b$ (member) defined as:

$$
\begin{aligned}
& m b(X,[Y \mid Y s]) \rightarrow \mathrm{t} \Leftarrow X \bowtie Y \\
& m b(X,[Y \mid Y s]) \rightarrow \mathrm{t} \Leftarrow m b(X, Y s) \bowtie \mathrm{t}
\end{aligned}
$$

If we try to evaluate the expression $m b(\operatorname{coin},[s(h)])$ it will fail. Intuitively, from definition of $h$ the list in the second argument can be reduced to lists of the form $[s(s(\ldots))]$ and the possible values of coin, $z$ and $s(z)$, do not belong to those lists. The $C R W L F$-calculus allows to build a proof for this fact, that is, $\operatorname{mb}(\operatorname{coin},[s(h)]) \triangleleft\{\mathrm{F}\}$, in the following way: by application of rule 4 the proof could proceed by generating $S A S$ 's for the arguments

$$
\operatorname{coin} \triangleleft\{z, s(z)\} \quad\left(\varphi_{1}\right) \quad[s(h)] \triangleleft\{[s(s(\perp))]\} \quad\left(\varphi_{2}\right)
$$

and then collecting the contributions of rules of $m b$ for each possible combination of values for the arguments; for the pair $(z,[s(s(\perp))])$ the contribution of the rules defining $m b$ (here we write $\triangleleft_{1}$ to refer to the first rule of $m b$ and $\triangleleft_{2}$ for the second) will be

$$
m b(z,[s(s(\perp))]) \triangleleft_{1}\{\mathrm{~F}\} \quad\left(\varphi_{3}\right) \quad m b(z,[s(s(\perp))]) \triangleleft_{2}\{\mathrm{~F}\} \quad\left(\varphi_{4}\right)
$$

and for the pair $(s(z),[s(s(\perp)])$ we will have

$$
m b(s(z),[s(s(\perp))]) \triangleleft_{1}\{\mathrm{~F}\} \quad\left(\varphi_{5}\right) \quad m b(s(z),[s(s(\perp))]) \triangleleft_{2}\{\mathrm{~F}\} \quad\left(\varphi_{6}\right)
$$

The full derivation takes the form:

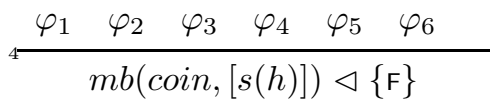

The $S A S\{\mathrm{~F}\}$ in the conclusion comes from the union of all the contributing $S A S$ 's of $\varphi_{3}, \varphi_{4}, \varphi_{5}$ and $\varphi_{6}$. The statements $\varphi_{1}$ to $\varphi_{6}$ require of course their own proof, which we describe now. At each step, we indicate by a number on the left the rule of the calculus applied in each case:

The derivation for $\varphi_{1}$ is not difficult to build, and for $\varphi_{2}$ it is: 


$$
\begin{aligned}
& z \triangleleft\{\perp\} \\
& s(z) \triangleleft\{s(\perp)\} \\
& h \triangleleft\{\perp\} \\
& s(h) \triangleleft\{s(\perp)\} \\
& h \triangleleft _ { 1 } \{ s ( \perp ) \} \quad 6 \longdiv { s \triangleleft _ { 2 } \{ s ( \perp ) \} } \\
& h \triangleleft\{s(\perp)\} \\
& s(h) \triangleleft\{s(s(\perp))\} \quad[] \triangleleft\{[]\} \\
& {[s(h)] \triangleleft\{[s(s(\perp))]\}}
\end{aligned}
$$

For $\varphi_{3}$ it can be done as follows:

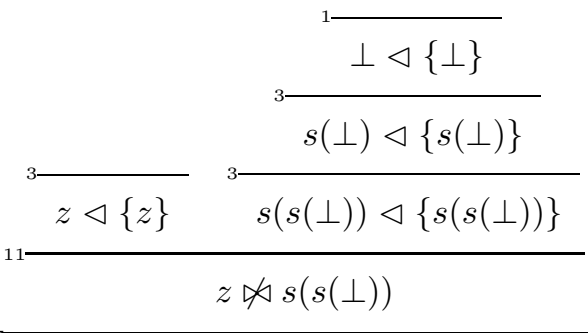

$$
\begin{aligned}
& \varphi_{3} \equiv m b(z,[s(s(\perp))]) \triangleleft_{1}\{\mathrm{~F}\}
\end{aligned}
$$

Here, the failure is due to a failure in the constraint $z \bowtie s(s(\perp))$ of the used program rule, what requires to prove the complementary constraint $z \not s s(s(\perp))$ by rule (11). In this case there is a clear clash of constructors $(z$ and $s)$.

For $\varphi_{4}$ a derivation might be this one:

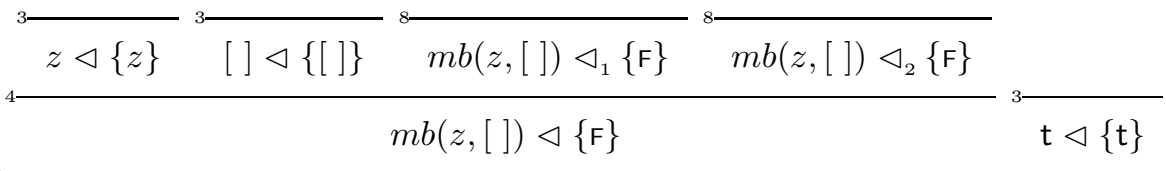

$$
\begin{aligned}
& m b(z,[]) \bowtie\{\mathrm{t}\} \\
& \varphi_{4} \equiv m b(z,[s(s(\perp))]) \triangleleft_{2}\{\mathrm{~F}\}
\end{aligned}
$$

The failure is due again to a failure in the constraint of the rule and in this case the complementary constraint is $m b(z,[]) \bowtie t$. Now it is involved the failure for the expression $m b(z,[])$ that is proved by rule (4) of the calculus. The $S A S$ 's for the arguments only produce the combination $(z,[])$ and both rules of $m b$ fail over it by rule (8) of the calculus.

The derivations for $\varphi_{5}$ and $\varphi_{6}$ are quite similar to those of $\varphi_{3}$ and $\varphi_{4}$ respectively. All the contributions obtained from $\varphi_{3}, \varphi_{4}, \varphi_{5}$ and $\varphi_{6}$ are $\{\mathrm{F}\}$, and putting them together we obtain $\{\mathrm{F}\}$ as an $S A S$ for the original expression $m b(\operatorname{coin},[s(h)])$, as it was expected. 


\section{Properties of $C R W L F$}

In this section we explore some technical properties of the $C R W L F$-calculus which are the key for proving the results of the next section, where we relate the $C R W L F$ calculus to the $C R W L$-calculus. In the following we assume a fixed program $\mathcal{P}$.

The non-determinism of the $C R W L F$-calculus allows to obtain different $S A S$ 's for the same expression. As an $S A S$ for an expression is a finite approximation to the denotation of the expression it is expected some kind of consistency between $S A S$ 's for the same expression. Given two of them, we cannot ensure that one $S A S$ must be more defined than the other in the sense that all the elements of the first are more defined than all of the second. For instance, two $S A S$ 's for coin are $\{\perp, s(z)\}$ and $\{z, \perp\}$. The kind of consistency for $S A S$ 's that we can expect is the following:

Definition 2 (Consistent Sets of c-terms)

Two sets $\mathcal{C}, \mathcal{C}^{\prime} \subseteq C$ Term $m_{\perp, \mathrm{F}}$ are consistent iff for all $t \in \mathcal{C}$ there exists $t^{\prime} \in \mathcal{C}^{\prime}$ (and vice versa, for all $t^{\prime} \in \mathcal{C}^{\prime}$ there exists $t \in \mathcal{C}$ ) such that $t$ and $t^{\prime}$ are consistent.

Our first result states that two different $S A S$ 's for the same expression must be consistent.

Theorem 1 (Consistency of $S A S$ )

Given $e \in$ Term $_{\perp, \mathrm{F}}$, if $\mathcal{P} \vdash_{C R W L F} e \triangleleft \mathcal{C}$ and $\mathcal{P} \vdash_{C R W L F} e \triangleleft \mathcal{C}^{\prime}$, then $\mathcal{C}$ and $\mathcal{C}^{\prime}$ are consistent.

This result is a trivial corollary of part $a$ ) of the following lemma.

Lemma 1 (Consistency)

For any $e, e^{\prime}, e_{1}, e_{2}, e_{1}^{\prime}, e_{2}^{\prime} \in T e r m_{\perp, \mathrm{F}}$

a) If $e, e^{\prime}$ are consistent, $\mathcal{P} \vdash_{C R W L F} e \triangleleft \mathcal{C}$ and $\mathcal{P} \vdash_{C R W L F} e^{\prime} \triangleleft \mathcal{C}^{\prime}$, then $\mathcal{C}$ and $\mathcal{C}^{\prime}$ are consistent.

b) If $e_{1}, e_{1}^{\prime}$ are consistent and $e_{2}, e_{2}^{\prime}$ are also consistent, then: $\mathcal{P} \vdash_{C R W L F} e_{1} \diamond e_{2} \Rightarrow$ $\mathcal{P} \nvdash_{C R W L F} e_{1}^{\prime} \tilde{\nabla} e_{2}^{\prime}$

Proof

For proving the consistency lemma we will split $b$ ) into $b .1), b .2$ ) and also strengthen the lemma with a new part $c)$ :

b) If $e_{1}, e_{1}^{\prime}$ are consistent and $e_{2}, e_{2}^{\prime}$ are also consistent, then:

b.1) $\mathcal{P} \vdash_{C R W L F} e_{1} \bowtie e_{2} \Rightarrow \mathcal{P} \nvdash_{C R W L F} e_{1}^{\prime} \bowtie e_{2}^{\prime}$

b.2) $\mathcal{P} \vdash_{C R W L F} e_{1} \diamond e_{2} \Rightarrow \mathcal{P} \forall_{C R W L F} e_{1}^{\prime} \phi e_{2}^{\prime}$

c) Given $\bar{t}, \bar{t}^{\prime} \in C T e r m_{\perp, \mathrm{F}} \times \ldots \times C T e r m_{\perp, \mathrm{F}}$ pairwise consistent and $R \in \mathcal{P}_{f}$, if $\mathcal{P} \vdash_{C R W L F}$ $f(\bar{t}) \triangleleft_{R} \mathcal{C}, f\left(\bar{t}^{\prime}\right) \triangleleft_{R} \mathcal{C}^{\prime}$, then $\mathcal{C}$ and $\mathcal{C}^{\prime}$ are consistent.

Now we will prove $a), b$ ) and $c$ ) simultaneously by induction on the size $l$ of the derivation for $e \triangleleft \mathcal{C}$ in $a), e_{1} \bowtie e_{2}$ in $\left.b .1\right), e_{1} \diamond e_{2}$ in $\left.b .2\right)$ and $f(\bar{t}) \triangleleft_{R} \mathcal{C}$ in $\left.c\right)$.

$\underline{l=1}$ :

a) The possible derivations in one step are: 
- $e \triangleleft\{\perp\}$. This $S A S$ is consistent with any other;

- $X \triangleleft\{X\}$. Then either $e^{\prime}=X$ or $e^{\prime}=\perp$, so the possibilities for $\mathcal{C}^{\prime}$ are $\{X\}$ or $\{\perp\}$, both consistent with $\{X\}$;

- $c \triangleleft\{c\}$, where $c \in D C^{0} \cup\{\mathrm{F}\}$. In this case $e^{\prime}$ must be $c$ or $\perp$, whose possible $S A S^{\prime}$ 's are $\{c\}$ and $\{\perp\}$, that are consistent with $\{c\}$.

b) There is no derivation of the form $e \bowtie e^{\prime}$ or $e \diamond e^{\prime}$ in one step.

c) The possible derivations of the form $f(\bar{t}) \triangleleft_{R} \mathcal{C}$ are:

- $f(\bar{t}) \triangleleft_{R}\{\perp\}$. This $S A S$ is consistent with any other;

- $f(\bar{t}) \triangleleft_{R}\{\mathrm{~F}\}$, by means of rule 8, i.e., there exists some $R \equiv(f(\bar{s}) \rightarrow e \Leftarrow \bar{C}) \in$ $\mathcal{P}_{f}$ and some $i$ such that $s_{i}$ and $t_{i}$ have a $D C \cup\{\mathrm{F}\}$-clash at some position $p$. The $S A S \mathcal{C}^{\prime}$ for $f\left(\bar{t}^{\prime}\right)$ using the function rule $R$ must be done by one of the rules 5 to 8 :

- if rule 5 is used then $\mathcal{C}^{\prime}=\{\perp\}$ that is consistent with $\mathcal{C}$;

- rule 6 is not applicable: $t_{i}$ and $t_{i}^{\prime}$ are consistent because $\bar{t}$ and $\bar{t}^{\prime}$ are pairwise consistent; then either $t_{i}^{\prime}$ at position $p$ has the same constructor symbol as $t_{i}$ (and then the clash with $s_{i}$ remains), or $t_{i}^{\prime}$ at $p$ or some of its ancestor positions has $\perp$. In both cases it is clear that there is not any c-instance of $R$ for using rule 6 ;

- by rules 7 or 8 the $S A S$ is $\{\mathrm{F}\}$ that is consistent with the initial one $\{\mathrm{F}\}$.

\section{$\underline{l \Rightarrow l+1}$}

a) In $l+1$ steps the possible derivations for $e \triangleleft \mathcal{C}$ are:

- $\frac{e_{1} \triangleleft \mathcal{C}_{1} \quad \ldots \quad e_{n} \triangleleft \mathcal{C}_{n}}{e=c\left(e_{1}, \ldots, e_{n}\right) \triangleleft\left\{c\left(t_{1}, \ldots, t_{n}\right) \mid \bar{t} \in \mathcal{C}_{1} \times \ldots \times \mathcal{C}_{n}\right\}}$ by rule 3 , where $c \in D C^{n}$ $(n>0)$. Then either $e^{\prime}=\perp$, whose only possible $S A S$ is $\{\perp\}$, that is consistent with any other, or $e^{\prime}=c\left(e_{1}^{\prime}, \ldots, e_{n}^{\prime}\right)$ with $e_{i}$ and $e_{n}^{\prime}$ being consistent for $i \in$ $\{1, \ldots, n\}$ and the $S A S$ is produced by rule 3 :

$$
\frac{e_{1}^{\prime} \triangleleft \mathcal{C}_{1}^{\prime} \quad \ldots \quad e_{n}^{\prime} \triangleleft \mathcal{C}_{n}^{\prime}}{e^{\prime}=c\left(e_{1}^{\prime}, \ldots, e_{n}^{\prime}\right) \triangleleft\left\{c\left(t_{1}^{\prime}, \ldots, t_{n}^{\prime}\right) \mid t^{\prime} \in \mathcal{C}_{1}^{\prime} \times \ldots \times \mathcal{C}_{n}^{\prime}\right\}}
$$

By i.h. $\mathcal{C}_{i}^{\prime}$ is consistent with $\mathcal{C}_{i}$ for all $i \in\{1, \ldots, n\}$ and then it is clear that $\mathcal{C}$ and $\mathcal{C}^{\prime}$ are also consistent.

- $\frac{e_{1} \triangleleft \mathcal{C}_{1} \quad \ldots \quad e_{n} \triangleleft \mathcal{C}_{n} \quad f(\bar{t}) \triangleleft_{R} \mathcal{C}_{R, \bar{t}}}{e=f\left(e_{1}, \ldots, e_{n}\right) \triangleleft \bigcup_{R \in \mathcal{P}_{f}, \bar{t} \in \mathcal{C}_{1} \times \ldots \times \mathcal{C}_{n}} \mathcal{C}_{R, \bar{t}}}$ by rule 4 . Then either $e^{\prime}=\perp$ whose only possible $S A S$ is $\{\perp\}$ that is consistent with any other, or $e^{\prime}=$ $f\left(e_{1}^{\prime}, \ldots, e_{n}^{\prime}\right)$ with $e_{i}, e_{i}^{\prime}$ consistent for all $i \in\{1, \ldots, n\}$. If the $S A S$ for $e^{\prime}$ is generated by rule 1 of the calculus, the result would be clear and for rule 4 we have $\frac{e_{1}^{\prime} \triangleleft \mathcal{C}_{1}^{\prime} \quad \ldots \quad e_{n}^{\prime} \triangleleft \mathcal{C}_{n}^{\prime} \quad f\left(\bar{t}^{\prime}\right) \triangleleft_{R} \mathcal{C}_{R, \bar{t}^{\prime}}}{e^{\prime}=f\left(e_{1}^{\prime}, \ldots, e_{n}^{\prime}\right) \triangleleft \bigcup_{R \in \mathcal{P}_{f}, \bar{t}^{\prime} \in \mathcal{C}_{1}^{\prime} \times \ldots \times \mathcal{C}_{n}^{\prime}} \mathcal{C}_{R, \bar{t}^{\prime}}}$

By i.h. $\mathcal{C}_{i}$ and $\mathcal{C}_{i}^{\prime}$ are consistent for all $i \in\{1, \ldots, n\}$, what means that for each $\bar{t} \in \mathcal{C}_{1} \times \ldots \times \mathcal{C}_{n}$ there exists $\bar{t}^{\prime} \in \mathcal{C}_{1}^{\prime} \times \ldots \times \mathcal{C}_{n}^{\prime}$ consistent with $\bar{t}$. Again 
by i.h. we have that each $S A S \mathcal{C}_{R, \bar{t}}$ is consistent with $\mathcal{C}_{R, \bar{t}^{\prime}}$ and it can be easily proved that $\mathcal{C}=\bigcup_{R \in \mathcal{P}_{f}, \bar{t} \in \mathcal{C}_{1} \times \ldots \times \mathcal{C}_{n}} \mathcal{C}_{R, \bar{t}}$ is then consistent with $\mathcal{C}^{\prime}=$ $\bigcup_{R \in \mathcal{P}_{f}, \bar{t}^{\prime} \in \mathcal{C}_{1}^{\prime} \times \ldots \times \mathcal{C}_{n}^{\prime}} \mathcal{C}_{R, \bar{t}^{\prime}}$

- $\frac{e_{1} \triangleleft\{\mathrm{F}\}}{e=\text { fails }\left(e_{1}\right) \triangleleft\{\text { true }\}}$ by rule 13 . If $e^{\prime}=\perp$ the result is clear, else $e^{\prime}=$ fails $\left(e_{1}^{\prime}\right)$. Then the $S A S$ for $e^{\prime}$ requires to obtain an $S A S \mathcal{C}^{\prime}$ for $e_{1}^{\prime}$. By i.h., $\mathcal{C}^{\prime}$ must be consistent with $\{\mathrm{F}\}$ what means that $\mathcal{C}^{\prime}=\{\mathrm{F}\}$ or $\mathcal{C}^{\prime}=\{\perp\}$. Then, the possible $S A S$ s sor $e^{\prime}$ are $\{\perp\}$ and $\{$ true $\}$ (by the same rule 13), both consistent with $\{$ true $\}$.

- $\frac{e_{1} \triangleleft \mathcal{C}_{1}}{e=\text { fails }\left(e_{1}\right) \triangleleft\{\text { false }\}}$ by rule 14 , such that there exists $t \in \mathcal{C}_{1}$ with $t \neq \perp$, $t \neq \mathrm{F}$. If $e^{\prime}=\perp$ the result is clear. Otherwise, if $e^{\prime}=$ fails $\left(e_{1}^{\prime}\right)$ the $S A S$ for $e^{\prime}$ must be obtained by one of the rules 1,13 or 14 . By rule 1 it would be $\{\perp\}$ consistent with any other one; rule 13 would need to obtain the $S A S\{\mathrm{~F}\}$ for $e_{1}^{\prime}$, but this is not possible because it must be consistent with $\mathcal{C}_{1}$ by i.h., so rule 13 is not applicable; and rule 14 would provide the $S A S\{$ false $\}$ for $e^{\prime}$, consistent with itself.

b.1) If we have a derivation for $e_{1} \bowtie e_{2}$ by rule 9, there exist two $S A S$ 's $\mathcal{C}_{e_{1}}$ and $\mathcal{C}_{e_{2}}$ such that $e \triangleleft \mathcal{C}_{e_{1}}, e_{2} \triangleleft \mathcal{C}_{e_{2}}$ and there exist $t \in \mathcal{C}_{e_{1}}, t^{\prime} \in \mathcal{C}_{e_{2}}$ with $t \downarrow t^{\prime}$.

Now, let $e_{1}^{\prime}, e_{2}^{\prime}$ be consistent with $e_{1}, e_{2}$ respectively, and assume that $e_{1}^{\prime} \bowtie e_{2}^{\prime}$ can be proved. We reason by contradiction. Since $e_{1}^{\prime} \bowtie e_{2}^{\prime}$ is provable, we can prove $e_{1}^{\prime} \triangleleft \mathcal{C}_{e_{1}^{\prime}}, e_{2}^{\prime} \triangleleft \mathcal{C}_{e_{2}^{\prime}}$ such that for all $s \in \mathcal{C}_{e_{1}^{\prime}}, s^{\prime} \in \mathcal{C}_{e_{2}^{\prime}}$ it will be $s \downarrow s^{\prime}$.

By i.h. $\mathcal{C}_{e_{1}}$ is consistent with $\mathcal{C}_{e_{1}^{\prime}}$, what implies that there exists $u \in \mathcal{C}_{e_{1}^{\prime}}$ consistent with $t$, and then there exists $v$ such that $v \sqsupseteq u, v \sqsupseteq t$. In a similar way, there exists $u^{\prime} \in \mathcal{C}_{e_{2}^{\prime}}$ consistent with $t^{\prime}$, so there exists $v^{\prime}$ such that $v^{\prime} \sqsupseteq u^{\prime}, v^{\prime} \sqsupseteq t^{\prime}$.

As $u \in \mathcal{C}_{e_{1}^{\prime}}$ and $u^{\prime} \in \mathcal{C}_{e_{2}^{\prime}}$ we would have $u \Downarrow u^{\prime}$; by monotonicity of $\downarrow$ we have $v \Downarrow v^{\prime}$, what implies $\neg\left(v \downarrow v^{\prime}\right)$. But monotonicity of $\downarrow$, together with $t \downarrow t^{\prime}, v \sqsupset t, v^{\prime} \sqsupset t^{\prime}$, implies $v \downarrow v^{\prime}$, what is a contradiction.

b.2) The case of $e_{1} \diamond e_{2}$ proceeds similarly to $b .1$ ), using in this case monotonicity of $\uparrow$ and $\%$.

c) In $l+1$ steps the possible derivations for $f(\bar{t}) \triangleleft_{R} \mathcal{C}$ where $R \equiv(f(\bar{s}) \rightarrow e \Leftarrow \bar{C})$, are:

- $\frac{e \theta \triangleleft \mathcal{C} \quad \bar{C} \theta}{f(\bar{t}) \triangleleft_{R} \mathcal{C}}$ by rule 6 , using the c-instance $R \theta\left(\theta \in C\right.$ Subst $\left._{\perp, \mathrm{F}}\right)$, such that $\bar{t}=\bar{s} \theta$.

The derivation $f\left(\bar{t}^{\prime}\right) \triangleleft_{R} \mathcal{C}^{\prime}$ must be done by one of the rules 5 to 8 :

— if $f\left(\bar{t}^{\prime}\right) \triangleleft_{R}\{\perp\}$ by rule 5 , it is clear that this $S A S$ is consistent with $\mathcal{C}$;

— if the derivation is done by rule 6 , it will have the form $\frac{e \theta^{\prime} \triangleleft \mathcal{C}^{\prime} \quad \bar{C} \theta^{\prime}}{f\left(\bar{t}^{\prime}\right) \triangleleft_{R} \mathcal{C}^{\prime}}$ using a c-instance $R \theta^{\prime}$ of $R$. In particular, we have $\bar{t}^{\prime}=\bar{s} \theta^{\prime}$ and we also had $\bar{t}=\bar{s} \theta$. As $\bar{t}$ and $\bar{t}^{\prime}$ are pairwise consistent, and $\operatorname{var}(e) \subseteq \operatorname{var}(\bar{s})$ it is not difficult to 
see that $e \theta$ and $e \theta^{\prime}$ must be consistent. Then by i.h. (part $\left.a\right)$ ) we deduce that $\mathcal{C}$ and $\mathcal{C}^{\prime}$ are consistent $S A S^{\prime}$ s.

- rule 7 is not applicable: suppose that we have the derivation $\frac{\widetilde{C_{i}} \theta^{\prime}}{f\left(\bar{t}^{\prime}\right) \triangleleft_{R}\{\mathrm{~F}\}}$ using a c-instance $R \theta^{\prime}$ of $R$ and $C_{i} \theta^{\prime}$ being a constraint of $\bar{C} \theta$. Analogously to the previous case, we have that both members of $C_{i} \theta^{\prime}$ are consistent with the corresponding ones of $C_{i} \theta$; as $C_{i} \theta$ is provable then by i.h. (part $\left.b\right)$ ), $\widetilde{C}_{i} \theta^{\prime}$ is not provable, what means that rule 7 cannot be applied.

- rule 8 is not applicable: there cannot be a pair of $\bar{t}^{\prime}$ and $\bar{s}$ with a $D C \cup\{\mathrm{F}\}$ clash because then the corresponding pair of $\bar{t}^{\prime}$ and $\bar{s} \theta=\bar{t}$ would have the same clash (the substitution $\theta$ cannot make disappear the clash).

- $\frac{\widetilde{C_{i}} \theta}{f(\bar{t}) \triangleleft_{R}\{\mathrm{~F}\}}$ by rule 7 , being $R \theta$ a c-instance of the rule $R$ such that $\bar{t}=\bar{s} \theta$. The derivation $f\left(\bar{t}^{\prime}\right) \triangleleft_{R} \mathcal{C}^{\prime}$ can be done by one of the rules 5 to 8 :

- by rule 5 , the $S A S$ is $\{\perp\}$ that is consistent with any other;

- it is not possible to use rule 6 because we would need to prove a constraint $C_{i} \theta^{\prime}$ of a c-instance $R \theta^{\prime}$ of $R$. As $\bar{s} \theta=\bar{t}$ and $\bar{s} \theta^{\prime}=\bar{t}^{\prime}$ are pairwise consistent and $\operatorname{var}\left(C_{i}\right) \subseteq \operatorname{var}(\bar{s})$, both members of $C_{i} \theta$ and $C_{i} \theta^{\prime}$ will be also consistent. Then by i.h. (part $b)$ ), as $\widetilde{C_{i}} \theta$ is provable, $C_{i} \theta^{\prime}$ will not be provable.

- if 7 or 8 applies we will have $\mathcal{C}^{\prime}=\{\mathrm{F}\}$ that is consistent with $\mathcal{C}=\{\mathrm{F}\}$ (in fact, 8 would not be applicable).

As a trivial consequence of part $b$ ) we have:

Corollary 1

$\mathcal{P} \vdash_{C R W L F} e \diamond e^{\prime} \Rightarrow \mathcal{P} \nvdash_{C R W L F} e \tilde{\nabla} e^{\prime}$, for all $e, e^{\prime} \in T e r m_{\perp, \mathrm{F}}$

This justifies indeed our description of $\not$ and $\phi$ as computable approximations to the negations of $\bowtie$ and $\diamond$.

Another desirable property of our calculus is monotonicity, that we can informally understand in this way: the information that can be extracted from an expression cannot decrease when we add information to the expression itself. This applies also to the case of constraints: if we can prove a constraint and we consider more defined terms in both sides of it, the resulting constraint must be also provable. Formally:

Proposition 2 (Monotonicity of CRWLF)

For $e, e^{\prime}, e_{1}, e_{2}, e_{1}^{\prime}, e_{2}^{\prime} \in \operatorname{Term}_{\perp, \mathrm{F}}$

a) If $e \sqsubseteq e^{\prime}$ and $\mathcal{P} \vdash_{C R W L F} e \triangleleft \mathcal{C}$, then $\mathcal{P} \vdash_{C R W L F} e^{\prime} \triangleleft \mathcal{C}$

b) If $e_{1} \sqsubseteq e_{1}^{\prime}, e_{2} \sqsubseteq e_{2}^{\prime}$ and $\mathcal{P} \vdash_{C R W L F} e_{1} \diamond e_{2}$ then $\mathcal{P} \vdash_{C R W L F} e_{1}^{\prime} \diamond e_{2}^{\prime}$, where $\diamond \in\{\bowtie, \bowtie, \diamond, \phi\}$ 
Proof

Again we need to strengthen the result with a new part $c$ )

c) Given $\bar{t}, \bar{t}^{\prime} \in C T e r m_{\perp, \mathrm{F}} \times \ldots \times C T e r m_{\perp, \mathrm{F}}$ such that $t_{i} \sqsubseteq t_{i}^{\prime}$ for all $i \in\{1, \ldots, n\}$ and $R \in \mathcal{P}_{f}$, if $f(\bar{t}) \triangleleft_{R} \mathcal{C}$ then $f\left(\bar{t}^{\prime}\right) \triangleleft_{R} \mathcal{C}$

We will prove parts $a$ ), $b$ ) and $c$ ) simultaneously by induction on the size $l$ of the derivation for $e \triangleleft \mathcal{C}$ in $a), e_{1} \diamond e_{2}$ in $b$ ) and $f(\bar{t}) \triangleleft_{R} \mathcal{C}$ in $c$ ):

$\underline{l=1}$ :

a) The derivation of $e \triangleleft \mathcal{C}$ in one step can be:

- $e \triangleleft\{\perp\}$, and it is clear that also $e^{\prime} \triangleleft\{\perp\}$

- $X \triangleleft\{X\}$ : then $e=e^{\prime}=X$

- $c \triangleleft\{c\}, c \in D C^{0}$ : then $e=e^{\prime}=c$

b) For $e_{1} \diamond e_{2}$ there are not possible derivations in one steps.

c) For $f(\bar{t}) \triangleleft_{R} \mathcal{C}$ the derivations can be:

- $f(\bar{t}) \triangleleft_{R}\{\perp\}$, using rule 5. Then for all $\bar{t}^{\prime}$ we have $f\left(\bar{t}^{\prime}\right) \triangleleft_{R}\{\perp\}$

- $f(\bar{t}) \triangleleft_{R}\{\mathrm{~F}\}$, using rule 8 . Then $R \equiv(f(\bar{s}) \rightarrow e \Leftarrow \bar{C})$ and $\bar{t}$ and $\bar{s}$ have a $D C$-clash at some position. If $\bar{t} \sqsubseteq \bar{t}^{\prime}$ then $\bar{t}^{\prime}$ and $\bar{s}$ have the same clash, and rule 8 allows to prove also $f\left(t^{\prime}\right) \triangleleft_{R}\{\mathrm{~F}\}$.

$\underline{l \Rightarrow l+1:}$

a) We distinguish three cases for the derivation of $e \triangleleft \mathcal{C}$ :

- $e=c\left(e_{1}, \ldots, e_{n}\right)$. Then the derivation of $e \triangleleft \mathcal{C}$ must use the rule 3 and take the form: $\frac{e_{1} \triangleleft \mathcal{C}_{1} \ldots e_{n} \triangleleft \mathcal{C}_{n}}{c\left(e_{1}, \ldots, e_{n}\right) \triangleleft\left\{c\left(t_{1}, \ldots, t_{n}\right) \mid \bar{t} \in \mathcal{C}_{1} \times \ldots \times \mathcal{C}_{n}\right\}}$ Since $e \sqsubseteq e^{\prime}, e^{\prime}$ must take the form $e^{\prime}=c\left(e_{1}^{\prime}, \ldots, e_{n}^{\prime}\right)$ with $e_{1} \sqsubseteq e_{1}^{\prime}, \ldots, e_{n} \sqsubseteq e_{n}^{\prime}$. By i.h. we have $e_{1}^{\prime} \triangleleft \mathcal{C}_{1}, \ldots, e_{n}^{\prime} \triangleleft \mathcal{C}_{n}$ and with the same rule 3 we can build a derivation for $c\left(\bar{e}^{\prime}\right) \triangleleft \mathcal{C}$.

- $e=f\left(e_{1}, \ldots, e_{n}\right)$. Then the derivation of $e \triangleleft \mathcal{C}$ must use rule 4 and take the form: $\frac{e_{1} \triangleleft \mathcal{C}_{1} \ldots e_{n} \triangleleft \mathcal{C}_{n} f(\bar{t}) \triangleleft_{R} \mathcal{C}_{R, \bar{t}}}{f\left(e_{1}, \ldots, e_{n}\right) \triangleleft \bigcup_{R \in \mathcal{P}_{f}, \bar{t} \in \mathcal{C}_{1} \times \ldots \times \mathcal{C}_{n}} \mathcal{C}_{R, \bar{t}}} e^{\prime}$ must take the form $e^{\prime}=$ $f\left(e_{1}^{\prime}, \ldots, e_{n}^{\prime}\right)$ with $e_{i} \sqsubseteq e_{i}^{\prime}$. By i.h. we will have $e_{1}^{\prime} \triangleleft \mathcal{C}_{1}, \ldots, e_{n}^{\prime} \triangleleft \mathcal{C}_{n}$ and then we have the same tuples $\bar{t}$, the same $S A S$ 's $\mathcal{C}_{R, \bar{t}}$ and finally the same $S A S$ for $f\left(\bar{e}^{\prime}\right)$.

- $e=$ fails $\left(e_{1}\right)$. Then $e^{\prime}=$ fails $\left(e_{1}^{\prime}\right)$. The derivation $e \triangleleft \mathcal{C}$ must be done by one of the rules 13 or 14 , that require to obtain an $S A S$ for $e_{1}$. By i.h. if $e_{1} \triangleleft \mathcal{C}_{1}$ then $e_{1}^{\prime} \triangleleft \mathcal{C}_{1}$ and then the same rule (and only that) is applicable to obtain the same $S A S$ for $e^{\prime}$, that will be $\{$ true $\}$ if rule 13 is applicable or $\{$ false $\}$ if rule 14 is applied.

b) The derivation $e_{1} \diamond e_{2}$ with $\diamond \in\{\bowtie, \downarrow, \diamond, \phi\}$ will be done by generating the $S A S$ 's $e_{1} \triangleleft \mathcal{C}_{1}$ and $e_{2} \triangleleft \mathcal{C}_{2}$. By i.h. we have $e_{1}^{\prime} \triangleleft \mathcal{C}_{1}, e_{2}^{\prime} \triangleleft \mathcal{C}_{2}$ and then it is clear that $e_{1}^{\prime} \diamond e_{2}^{\prime}$ is also provable. 
c) We distinguish the following cases according to the rule used for the derivation of $f(\bar{t}) \triangleleft_{R} \mathcal{C}:$

- By rule 6 the derivation would be: $\frac{e \theta \triangleleft \mathcal{C} \bar{C} \theta}{f\left(t_{1}, \ldots, t_{n}\right) \triangleleft_{R} \mathcal{C}}$ where the rule $R$ is $R \equiv\left(f\left(s_{1}, \ldots, s_{n}\right) \rightarrow e \Leftarrow \bar{C}\right)$ and $\theta \in S u b s t_{\perp, \mathrm{F}}$ such that $\bar{s} \theta=\bar{t}$.

We will show that the same rule 6 is applicable for generating an $S A S$ for $f\left(\bar{t}^{\prime}\right)$ being $t_{i} \sqsubseteq t_{i}^{\prime}$ for all $i \in\{1, \ldots, n\}$. The idea is that if $t_{i} \sqsubseteq t_{i}^{\prime}$ then $t_{i}^{\prime}$ is the result of replacing some subterms $\perp$ of $t_{i}$ by c-terms more defined than $\perp$. As $s_{i} \in C T e r m$ then the corresponding positions or some ancestors must have variables in $s_{i}$. Then we can get a substitution $\theta^{\prime} \in C S u b s t_{\perp, \mathrm{F}}$ such that $\theta \sqsubseteq \theta^{\prime}$ and $s_{i} \theta^{\prime}=t_{i}^{\prime}$. A formal justification of this fact may be done by induction on the syntactic structure of $t_{i}$ and, as $\bar{s}$ is a linear tuple, the result can be extended in such a way that $\overline{s_{i}} \theta^{\prime}=\bar{t}^{\prime}$.

We also have that $e \theta \sqsubseteq e \theta^{\prime}$, so by i.h. we have $e \theta^{\prime} \triangleleft \mathcal{C}$. As the constraints $\bar{C} \theta$ are provable and $\theta \sqsubset \theta^{\prime}$, then by i.h. $b$ ), the constraints $\bar{C} \theta^{\prime}$ will also be provable. So we can build a derivation for $f\left(\bar{t}^{\prime}\right) \triangleleft_{R} \mathcal{C}$ by rule 6 .

- By rule 7 the derivation would be: $\frac{\widetilde{C}_{i} \theta}{f(\bar{t}) \triangleleft_{R}\{\mathrm{~F}\}}$ where the rule $R$ is $R \equiv$ $\left(f(\bar{s}) \rightarrow e \Leftarrow C_{1}, \ldots, C_{n}\right), i \in\{1, \ldots, n\}$ and $\theta \in C S u b s t_{\perp, \mathrm{F}}$ is such that $\bar{s} \theta=\bar{t}$. As $\bar{t} \sqsubseteq \bar{t}^{\prime}$, in a similar way as before there exists $\theta^{\prime}$ such that $\bar{s} \theta^{\prime}=\bar{t}^{\prime}$ and by i.h. we can prove $\widetilde{C}_{i} \theta^{\prime}$, what implies that we can build the derivation for $f\left(\bar{t}^{\prime}\right) \triangleleft_{R}\{\mathrm{~F}\}$, using rule 7 .

Remark: Monotonicity, as stated in Prop. 2] refers to the degree of evaluation of expressions and does not contradict the well known fact that negation as failure is a non-monotonic reasoning rule. In our setting it is also clearly true that, if we 'define more' the functions (i.e, we refine the program, by adding new rules to it), an expression can become reducible when it was previously failed.

The next property says that what is true for free variables is also true for any possible (totally defined) value, i.e., provability in $C R W L F$ is closed under total substitutions.

Proposition 3

For any $\theta \in C$ Subst, $e, e^{\prime} \in$ Term $_{\perp, \mathrm{F}}$

a) $\mathcal{P} \vdash_{\text {CRWLF }} e \triangleleft \mathcal{C} \Rightarrow \mathcal{P} \vdash_{\text {CRWLF }}$ e $\theta \triangleleft \mathcal{C} \theta$

b) $\mathcal{P} \vdash_{C R W L F} e \diamond e^{\prime} \Rightarrow \mathcal{P} \vdash_{C R W L F} e \theta \diamond e^{\prime} \theta$

Proof

Again we need to strengthen the result, with a new part $c$ ):

c) $f(\bar{t}) \triangleleft_{R} \mathcal{C} \Rightarrow f(\bar{t}) \theta \triangleleft_{R} \mathcal{C} \theta$, for any $\bar{t} \in C T e r m_{\perp, \mathrm{F}} \times \ldots \times C T e r m_{\perp, \mathrm{F}}$

We prove simultaneously the three parts by induction on the size $l$ of the derivations. 
$\underline{l=1}$ : in one step we can have the derivations $e \triangleleft\{\perp\}, c \triangleleft\{c\}\left(c \in D C^{0} \cup\{\mathrm{F}\}\right)$ and $X \triangleleft\{X\}$. The property is obvious for the first two and the third follows from the fact that if $t \in C T e r m_{\perp, F}$ then $t \triangleleft\{t\}$ is provable (this can be proved by induction on the depth of the term $t$ ). Notice that $X \theta \in C$ Term $\subset C T e r m_{\perp, \mathrm{F}}$, so $X \theta \triangleleft\{X \theta\}$.

$\underline{l \Rightarrow l+1}$ : now we can have the following derivations:

- by rule 3 we have $\frac{e_{1} \triangleleft \mathcal{C}_{1} \quad \ldots \quad e_{n} \triangleleft \mathcal{C}_{n}}{c\left(e_{1}, \ldots, e_{n}\right) \triangleleft\left\{c\left(t_{1}, \ldots, t_{n}\right) \mid \bar{t} \in \mathcal{C}_{1} \times \ldots \times \mathcal{C}_{n}\right\}}$ By i.h. we have $e_{i} \theta \triangleleft \mathcal{C}_{i} \theta$ for all $i \in\{1, \ldots, n\}$ and again by rule 3 we can build a derivation for $c(\bar{e}) \theta \triangleleft\left\{c\left(t_{1}, \ldots, t_{n}\right) \theta \mid \bar{t} \theta \in \mathcal{C}_{1} \theta \times \ldots \times \mathcal{C}_{n} \theta\right\}$

- by rule 4 we have $\frac{e_{1} \triangleleft \mathcal{C}_{1} \quad \ldots \quad e_{n} \triangleleft \mathcal{C}_{n} \quad \ldots \quad f(\bar{t}) \triangleleft_{R} \mathcal{C}_{R, \bar{t}} \quad \ldots}{f\left(e_{1}, \ldots, e_{n}\right) \triangleleft \bigcup_{R \in \mathcal{P}_{f}, \bar{t} \in \mathcal{C}_{1} \times \ldots \times \mathcal{C}_{n}} \mathcal{C}_{R, \bar{t}}}$ By i.h. we have $e_{i} \theta \triangleleft \mathcal{C}_{i} \theta$ for all $i \in\{1, \ldots, n\}$ and $f(\bar{t}) \theta \triangleleft_{R} \mathcal{C}_{R, \bar{t}} \theta$ for each $\left.\bar{t} \theta \in \mathcal{C}_{1} \theta \times \ldots \times \mathcal{C}_{n} \theta\right\}$ and each rule $R \in \mathcal{P}_{f}$. So we can get a derivation for $f(\bar{e}) \theta \triangleleft \bigcup_{R \in \mathcal{P}_{f}, \bar{t} \theta \in \mathcal{C}_{1} \theta \times \ldots \times \mathcal{C}_{n} \theta} \mathcal{C}_{R, \bar{t} \theta}$

- by rule 5 we have $\frac{}{f(\bar{t}) \triangleleft_{R}\{\perp\}}$ and it is clear $f(\bar{t}) \theta \triangleleft_{R}\{\perp\}$

- by rule 6 we have $\frac{e \theta^{\prime} \triangleleft \mathcal{C} \quad \bar{C} \theta^{\prime}}{f\left(\bar{t} \triangleleft_{R} \mathcal{C}\right.}$ where $(f(\bar{s}) \rightarrow e \Leftarrow \bar{C}) \in R$ and $\theta^{\prime} \in$ CSubst $_{\perp, \mathrm{F}}$ is such that $f(\bar{t})=f(\bar{s}) \theta^{\prime}$. For the call $f(\bar{t}) \theta$ we can get the appropriate c-instance by composing $\theta^{\prime}$ and $\theta$, so $f(\bar{t}) \theta=f(\bar{s}) \theta^{\prime} \theta$. By i.h. we have $e \theta^{\prime} \theta \triangleleft \mathcal{C} \theta$ and $\bar{C} \theta^{\prime} \theta$, and then $f(\bar{t}) \theta \triangleleft_{R} \mathcal{C} \theta$ by the same rule 6 .

- by rule 7 we have $\frac{e_{i} \theta^{\prime} \tilde{\diamond} e_{i}^{\prime} \theta^{\prime}}{f(\bar{t}) \triangleleft_{R}\{F\}}$ where $(f(\bar{s}) \rightarrow e \Leftarrow \bar{C}) \in R$ and $\theta^{\prime} \in$ CSubst $_{\perp, \mathrm{F}}$ is such that $f(\bar{t})=f(\bar{s}) \theta^{\prime}$. As before, for the call $f(\bar{t}) \theta$ we can get the appropriate c-instance by composing $\theta^{\prime}$ and $\theta$, so $f(\bar{t}) \theta=f(\bar{s}) \theta^{\prime} \theta$. By i.h. we have $e_{i} \theta^{\prime} \theta \tilde{\nabla} e_{i}^{\prime} \theta^{\prime} \theta$, and then $f(\bar{t}) \theta \triangleleft_{R}\{F\}$

- by rule 8 we have $\frac{}{f\left(t_{1}, \ldots, t_{n}\right) \triangleleft_{R}\{F\}}$ where $R \equiv\left(f\left(s_{1}, \ldots, s_{n}\right) \rightarrow e \Leftarrow \bar{C}\right)$ and such that $t_{i}$ and $s_{i}$ have a $D C \cup\{\mathrm{F}\}$-clash for some $i \in\{1, \ldots, n\}$. It is clear that $t_{i} \theta$ and $s_{i}$ will have the same clash so $f(\bar{t}) \theta \triangleleft_{r}\{\mathrm{~F}\}$

- by rules 9 to 12 , the derivation would have the form $e \diamond e^{\prime}$. By i.h. we have $e \theta \triangleleft$ $\mathcal{C} \theta \quad e^{\prime} \theta \triangleleft \mathcal{C}^{\prime} \theta$. Now, if we take $t \in \mathcal{C}, t^{\prime} \in \mathcal{C}^{\prime}$ and $t \Re t^{\prime}$ holds (where $\Re \in\{\downarrow, \uparrow, \downarrow, \downarrow\}$ ), then $t \theta \Re t^{\prime} \theta$ also holds, by Prop. 1 It follows that $e \theta \diamond e^{\prime} \theta$.

- by rule 13 (or rule 14), it must be $e=f a i l s\left(e_{1}\right)$. This rule requires to obtain an $S A S$ for $e_{1}$, say $e_{1} \triangleleft \mathcal{C}_{1}$. Then by i.h. $e_{1} \theta \triangleleft \mathcal{C}_{1} \theta$ and it is clear that rule 13 will be applicable to derive $e \triangleleft\{$ true $\}$ (or $e \triangleleft\{$ false $\}$ by rule 14).

\section{$6 C R W L F$ related to $C R W L$}

The $C R W L F$-calculus has been built as an extension of $C R W L$ for dealing with failure. Here we show that our aims have been achieved with respect to these two 
emphasized aspects. In order to establish the relations between both calculus we consider in this section the class of programs defined for $C R W L$, i.e., rules cannot use the function fails. This means that rules 13 and 14 of the $C R W L F$-calculus are not considered here.

First, we show that the $C R W L F$-calculus indeed extends $C R W L$. Parts $a$ ) and $b$ ) of the next result show that statements $e \triangleleft \mathcal{C}$ generalize approximation statements $e \rightarrow t$ of $C R W L$. Parts $c$ ) and $d$ ) show that $C R W L F$ and $C R W L$ are able to prove exactly the same joinabilities and divergences (if $\mathrm{F}$ is ignored for the comparison).

Proposition 4

For any $e, e^{\prime} \in$ Term $_{\perp, \mathrm{F}}$

a) $\mathcal{P} \vdash_{C R W L F} e \triangleleft \mathcal{C} \Rightarrow \forall t \in \mathcal{C}, \mathcal{P} \vdash_{C R W L} \hat{e} \rightarrow \hat{t}$

b) $\mathcal{P} \vdash_{C R W L} \hat{e} \rightarrow t \Rightarrow \exists \mathcal{C}$ such that $t \in \mathcal{C}$ and $\mathcal{P} \vdash_{C R W L F} e \triangleleft \mathcal{C}$

c) $\mathcal{P} \vdash_{C R W L F} e \bowtie e^{\prime} \Leftrightarrow \mathcal{P} \vdash_{C R W L} \hat{e} \bowtie \hat{e}^{\prime}$

d) $\mathcal{P} \vdash_{C R W L F} e \diamond e^{\prime} \Leftrightarrow \mathcal{P} \vdash_{C R W L} \hat{e} \diamond \hat{e}^{\prime}$

In order to prove the property we split it into two separate lemmas. The first one contains $a$ ), the right implication of $c$ ) and $d$ ) and a new part $e$ ):

\section{Lemma 2}

Let $\mathcal{P}$ a $C R W L F$-program. Then:

a) $\mathcal{P} \vdash_{C R W L F} e \triangleleft \mathcal{C} \Rightarrow \forall t \in \mathcal{C}, \mathcal{P} \vdash_{C R W L} \hat{e} \rightarrow \hat{t}$

c) $\mathcal{P} \vdash_{C R W L F} e \bowtie e^{\prime} \Rightarrow \mathcal{P} \vdash_{C R W L} \hat{e} \bowtie \hat{e}^{\prime}$

d) $\mathcal{P} \vdash_{C R W L F} e \diamond e^{\prime} \Rightarrow \mathcal{P} \vdash_{C R W L} \hat{e} \diamond \hat{e}^{\prime}$

e) Given $\bar{t} \in C$ Term $_{\perp, \mathrm{F}} \times \ldots \times C$ Term $_{\perp, \mathrm{F}}$ and $R \in \mathcal{P}_{f}: \mathcal{P} \vdash_{C R W L F} f(\bar{t}) \triangleleft_{R} \mathcal{C} \Rightarrow$ $\forall t \in \mathcal{C}, \mathcal{P} \vdash_{C R W L} \widehat{f(\bar{t})} \rightarrow \hat{t}$

Proof

We prove simultaneously all the parts by induction on the size $l$ of the corresponding derivation:

$\underline{l=1}$ : the derivation can be:

- $\mathcal{P} \vdash_{C R W L F} e \triangleleft\{\perp\}$, and we have $\mathcal{P} \vdash_{C R W L} \hat{e} \rightarrow \perp$

- $\mathcal{P} \vdash_{C R W L F} X \triangleleft\{X\}$, we have $\hat{X}=X$ and $\mathcal{P} \vdash_{C R W L} X \rightarrow X$

- $\mathcal{P} \vdash_{C R W L F} c \triangleleft\{c\}$, where $c \in D C^{0}$ and we have $\hat{c}=c$ and $\mathcal{P} \vdash_{C R W L} c \rightarrow c$

- $\mathcal{P} \vdash_{C R W L F} \mathrm{~F} \triangleleft\{\mathrm{F}\}$, we have $\hat{\mathrm{F}}=\perp$ and $\mathcal{P} \vdash_{C R W L} \perp \rightarrow \perp$

- $\mathcal{P} \vdash_{C R W L F} f(\bar{t}) \triangleleft_{R}\{\perp\}$, and we have $\mathcal{P} \vdash_{C R W L} \widehat{f(\bar{t})} \rightarrow \perp$

- $\mathcal{P} \vdash_{C R W L F} f(\bar{t}) \triangleleft_{R}\{\mathrm{~F}\}$, and we have $\mathcal{P} \vdash_{C R W L} \widehat{f(\bar{t})} \rightarrow \perp$

$\underline{l \Rightarrow l+1}$ : the derivation can be:

- $\mathcal{P} \vdash_{C R W L F} c\left(e_{1}, \ldots, e_{n}\right) \triangleleft\left\{\ldots, c\left(t_{1}, \ldots, t_{n}\right), \ldots\right\}$, then by the rule 3 of $C R W L F$, it must be $\mathcal{P} \vdash_{C R W L F} e_{i} \triangleleft\left\{\ldots, t_{i}, \ldots\right\}$. By i.h. we have $\mathcal{P} \vdash_{C R W L} \hat{e}_{i} \rightarrow \hat{t}_{i}$ and then we can build the derivation $\mathcal{P} \vdash_{C R W L} c\left(\widehat{e_{1}, \ldots, e_{n}}\right) \rightarrow c\left(\widehat{t_{1}, \ldots, t_{n}}\right)$, by the rule 3 of $C R W L$. 
- $\mathcal{P} \vdash_{C R W L F} f\left(e_{1}, \ldots, e_{n}\right) \triangleleft\{\ldots, t, \ldots\}$. This derivation must use the rule 4 of $C R W L F$, and then we will have the derivations $\mathcal{P} \vdash_{C R W L F} e_{i} \triangleleft \mathcal{C}_{i}$ for all $i \in\{1, \ldots, n\}$ and $f(\bar{t}) \triangleleft_{R} \mathcal{C}_{R, \bar{t}}$. It must be $t \in \mathcal{C}_{R, \bar{t}}$ for some $\bar{t} \in \mathcal{C}_{1} \times \ldots \times \mathcal{C}_{n}$ and $R \in \mathcal{P}_{f}$. By i.h. we will have $\mathcal{P} \vdash_{C R W L} \widehat{f(\bar{t})} \rightarrow \hat{t}$

- $\mathcal{P} \vdash_{C R W L F} f(\bar{t}) \triangleleft_{R} \mathcal{C}$ by rule 6 of $C R W L F$, for which we take $R \equiv(f(\bar{s}) \rightarrow e \Leftarrow$ $\bar{C}), \theta \in C S u b s t_{\perp, \mathrm{F}}$ such that $\bar{s} \theta=\bar{t}$. We can define $\theta^{\prime} \in C S u b s t_{\perp}$ as $X \theta^{\prime}=\perp$ if $X \theta=\mathrm{F}$ and $X \theta^{\prime}=X \theta$, in other case. So we have $\bar{s} \theta^{\prime}=\hat{\bar{t}}, e \theta^{\prime}=\widehat{e \theta}$ and $\bar{C} \theta^{\prime}=\widehat{\bar{C}}$. Now we can take $(f(\bar{s}) \rightarrow e \Leftarrow \bar{C}) \theta^{\prime} \in[\mathcal{P}]_{\perp}$. We also have $e \theta \triangleleft \mathcal{C}$ and if $t \in \mathcal{C}$ by i.h. we have $\widehat{e \theta} \rightarrow \hat{t}$, or what is the same, $e \theta^{\prime} \rightarrow \hat{t}$. Also by i.h. $\bar{C} \theta^{\prime}=\widehat{\bar{C}} \theta$ is provable within $C R W L$, and therefore $f(\bar{t}) \rightarrow \hat{t}$ by rule 4 of $C R W L$.

- $\mathcal{P} \vdash_{C R W L F} f(\bar{t}) \triangleleft_{R}\{\mathrm{~F}\}$ and we have $\mathcal{P} \vdash_{C R W L} \widehat{f(\bar{t})} \rightarrow \perp$

- $\mathcal{P} \vdash_{C R W L F} e \bowtie e^{\prime}$ using the rule 9 of $C R W L F$. Then we will have $\mathcal{P} \vdash_{C R W L F} e \triangleleft \mathcal{C}$, $\mathcal{P} \vdash_{\text {CRWLF }} e^{\prime} \triangleleft \mathcal{C}^{\prime}$ and there exist $t \in \mathcal{C}, t^{\prime} \in \mathcal{C}^{\prime}$ such that $t \downarrow t^{\prime}$ (by definition of $\downarrow$ it is easy to see that $t=t^{\prime}$ ). By i.h. we have $\mathcal{P} \vdash_{C R W L} \hat{e} \rightarrow \hat{t}$ and $\mathcal{P} \vdash_{C R W L} \hat{e}^{\prime} \rightarrow \hat{t}$ and by rule 5 of $C R W L$ we have $\mathcal{P} \vdash_{C R W L} \hat{e} \bowtie \hat{t}$.

- $\mathcal{P} \vdash_{C R W L F} e \diamond e^{\prime}$ using the rule 10 of $C R W L F$. Then we will have $\mathcal{P} \vdash_{C R W L F} e \triangleleft \mathcal{C}$, $\mathcal{P} \vdash_{C R W L F} e^{\prime} \triangleleft \mathcal{C}^{\prime}$ and there exist $t \in \mathcal{C}, t^{\prime} \in \mathcal{C}^{\prime}$ such that $t \uparrow t^{\prime}$. By definition of $\uparrow, t$ and $t^{\prime}$ have a $D C$-clash. By i.h. we have $\mathcal{P} \vdash_{C R W L} \hat{e} \rightarrow \hat{t}$ and $\mathcal{P} \vdash_{C R W L} \hat{e}^{\prime} \rightarrow \hat{t}$ and by rule 5 of $C R W L$ we have $\mathcal{P} \vdash_{C R W L} \hat{e} \diamond \hat{t}$.

We now state the second lemma for Proposition 4 in which the part $b$ ) and the left implications of $c$ ) and $d$ ) will be proved.

\section{Lemma 3}

For any $e, e^{\prime} \in$ Term $_{\perp, \mathrm{F}}$

b) $\mathcal{P} \vdash_{C R W L} \hat{e} \rightarrow t \Rightarrow \exists \mathcal{C}$ such that $t \in \mathcal{C}$ and $\mathcal{P} \vdash_{C R W L F} e \triangleleft \mathcal{C}$

c) $\mathcal{P} \vdash_{C R W L} \hat{e} \bowtie \hat{e}^{\prime} \Rightarrow \mathcal{P} \vdash_{C R W L F} e \bowtie e^{\prime}$

d) $\mathcal{P} \vdash_{C R W L} \hat{e} \diamond \hat{e}^{\prime} \Rightarrow \mathcal{P} \vdash_{C R W L F} e \diamond e^{\prime}$

Proof

We prove the three parts simultaneously by induction on the size $l$ of the derivation: $\underline{l=1}$ : the derivation can be:

- $\mathcal{P} \vdash_{C R W L} \hat{e} \rightarrow \perp$ and it is clear that $\mathcal{P} \vdash_{C R W L F} e \triangleleft\{\perp\}$

- $\mathcal{P} \vdash_{C R W L} X \rightarrow X$ and it is clear that $\mathcal{P} \vdash_{C R W L F} X \triangleleft\{X\}$

- $\mathcal{P} \vdash_{C R W L} c \rightarrow c$ with $c \in D C^{0}$ and it is clear that $\mathcal{P} \vdash_{C R W L F} c \triangleleft\{c\}$

$l \Rightarrow l+1$ : the derivation can be of the following four forms:

- $\mathcal{P} \vdash_{C R W L} c\left(\widehat{e_{1}, \ldots,}, e_{n}\right) \rightarrow c\left(t_{1}, \ldots, t_{n}\right)$ by rule 3 of $C R W L$ and then we have $\mathcal{P} \vdash_{C R W L}$ $\hat{e}_{i} \rightarrow t_{i}$ for all $i \in\{1, \ldots n\}$. By i.h. we have $\mathcal{P} \vdash_{C R W L F} e_{i} \triangleleft \mathcal{C}_{i}$ with $t_{i} \in \mathcal{C}_{i}$ and by rule 3 of $C R W L F$ we have $\mathcal{P} \vdash_{C R W L F} c\left(e_{1}, \ldots, e_{n}\right) \triangleleft \mathcal{C}$ with $c\left(t_{1}, \ldots, t_{n}\right) \in \mathcal{C}$.

- $\mathcal{P} \vdash_{C R W L} f\left(\widehat{e_{1}, \ldots, e_{n}}\right) \rightarrow t$, then there must exist a rule $R=(f(\bar{s}) \rightarrow e \Leftarrow \bar{C}) \in \mathcal{P}$ and $\theta \in C S u b s t_{\perp}$ such that by rule 4 of $C R W L$ we will have the derivation

$$
\frac{\hat{e_{1}} \rightarrow s_{1} \theta \quad \ldots \quad \hat{e_{n}} \rightarrow s_{n} \theta \quad e \theta \rightarrow t \quad \bar{C} \theta}{f\left(\widehat{e_{1}, \ldots,} e_{n}\right) \rightarrow t} \text { By i.h. we have: }
$$


i) there exists $\mathcal{C}_{i}$ such that $\mathcal{P} \vdash_{C R W L F} e_{i} \triangleleft \mathcal{C}_{i}$ with $s_{i} \theta \in \mathcal{C}_{i}$

ii) there exists $\mathcal{C}^{\prime}$ such that $\mathcal{P} \vdash_{C R W L F} e \theta \triangleleft \mathcal{C}^{\prime}$ with $t \in \mathcal{C}^{\prime}$

iii) $\mathcal{P} \vdash_{C R W L F} \bar{C} \theta$

From $i$ i) and iii), by rule 6 of $C R W L F$ we can build the derivation $\mathcal{P} \vdash_{C R W L F}$ $f\left(s_{i} \theta\right) \triangleleft_{R} \mathcal{C}^{\prime}$ using the c-instance $R \theta$. With this derivation and $i$ ) we have $\mathcal{P} \vdash_{C R W L F}$ $f(\bar{e}) \triangleleft \mathcal{C}$ such that $\mathcal{C}^{\prime} \subseteq \mathcal{C}$, so $t \in \mathcal{C}$.

- $\mathcal{P} \vdash_{C R W L} \hat{e} \bowtie \hat{e}^{\prime}$, using the rule 5 of $C R W L$. It follows that $\mathcal{P} \vdash_{C R W L} \hat{e} \rightarrow t$ and $\mathcal{P} \vdash_{C R W L} \hat{e}^{\prime} \rightarrow t$ for some $t \in C T$ erm. By i.h. $\mathcal{P} \vdash_{C R W L F} e \triangleleft \mathcal{C}$ and $\mathcal{P} \vdash_{C R W L F} e^{\prime} \triangleleft \mathcal{C}^{\prime}$ where $t \in \mathcal{C} \cap \mathcal{C}^{\prime}$. Taking into account that $t \downarrow t$ for all $t \in C$ Term, by rule 9 of $C R W L F$ we can build a derivation for $\mathcal{P} \vdash_{C R W L F} e \bowtie e^{\prime}$.

- $\mathcal{P} \vdash_{C R W L} \hat{e} \diamond \hat{e}^{\prime}$, using the rule 6 of $C R W L$. It follows that $\mathcal{P} \vdash_{C R W L} \hat{e} \rightarrow t$ and $\mathcal{P} \vdash_{C R W L} \hat{e}^{\prime} \rightarrow t^{\prime}$ where $t, t^{\prime} \in C T e r m_{\perp}$ and have a $D C$-clash. By i.h. $\mathcal{P} \vdash_{C R W L F} e \triangleleft \mathcal{C}$ and $\mathcal{P} \vdash_{C R W L F} e^{\prime} \triangleleft \mathcal{C}^{\prime}$ where $t \in \mathcal{C}$ and $t^{\prime} \in \mathcal{C}^{\prime}$. By definition of $\uparrow$ and by rule 10 of $C R W L F$ we can build a derivation for $\mathcal{P} \vdash_{C R W L F} e \diamond e^{\prime}$.

All the previous results make easy the task of proving that we have done things right with respect to failure. We will need a result stronger than Prop. 4 which does not provide enough information about the relation between the denotation of an expression and each of its calculable $S A S$ 's.

\section{Proposition 5}

Given $e \in \operatorname{Term}_{\perp, \mathrm{F}}$, if $\mathcal{P} \vdash_{C R W L F} e \triangleleft \mathcal{C}$ and $\mathcal{P} \vdash_{C R W L} \hat{e} \rightarrow t$, then there exists $s \in \mathcal{C}$ such that $s$ and $t$ are consistent.

\section{Proof}

Assume $\mathcal{P} \vdash_{C R W L F} e \triangleleft \mathcal{C}$ and $\mathcal{P} \vdash_{C R W L} \hat{e} \rightarrow t$. By part $b$ ) of Prop. 4 there exists $\mathcal{C}^{\prime}$ such that $\mathcal{P} \vdash_{C R W L F} e \triangleleft \mathcal{C}^{\prime}$ with $t \in \mathcal{C}^{\prime}$.

By Theorem 1 it follows that $\mathcal{C}$ and $\mathcal{C}^{\prime}$ are consistent. By definition of consistent $S A S^{\prime}$ s, as $t \in \mathcal{C}^{\prime}$, then there exist $s \in \mathcal{C}$ such that $t$ and $s$ are consistent.

We easily arrive now at our final result.

Theorem 2

Given $e \in T e r m_{\perp, F}$, if $\mathcal{P} \vdash_{C R W L F} e \triangleleft\{\mathrm{F}\}$ then $\llbracket \hat{e} \rrbracket=\{\perp\}$

\section{Proof}

Assume $t \in \llbracket \hat{e} \rrbracket$. This means that $\mathcal{P} \vdash_{C R W L} \hat{e} \rightarrow t$, which in particular implies $t \in C$ Term $_{\perp}$. On the other hand, since $\mathcal{P} \vdash_{C R W L F} e \triangleleft\{\mathrm{F}\}$, we know from Prop. [5 that $\mathrm{F}$ and $t$ must be consistent. As $\mathrm{F}$ is consistent only with $\perp$ and itself, and $t \in C$ Term $_{\perp}$, we conclude that $t=\perp$. 


\section{Final Discussion and Future Work}

We have investigated how to deduce negative information from a wide class of functional logic programs. This is done by considering failure of reduction to head normal form, a notion playing a similar role, in the FLP setting, to that of negation as failure in logic programming, but having quite a different starting point. Negation as failure in $L P$ can be seen mainly as an operational idea (existence of a finite, failed search tree) of which a logical interpretation can be given (successful negated atoms are logical consequences of the completion of the program). The operational view of negation leads to an immediate implementation technique for negation included in all Prolog systems: to solve the negation of a goal, try to solve the goal and succeed if this attempt ends in failure. Unfortunately, as it is well-known, this implementation of negation is logically sound only for ground goals (see e.g. Apt and Bol (1994)).

Our approach has been different: we have given a logical status to failure by proposing the proof calculus CRWLF (Constructor based ReWriting Logic with Failure), which allows to deduce failure of reduction within $C R W L$ (González et al. 1996 González et al. 1999), a well established theoretical framework for FLP.

We must emphasize the fact that $C R W L F$ is not an operational mechanism for executing programs using failure, but a deduction calculus fixing the logical meaning of such programs. Exactly the same happens in González et al. 1996. González et al. 1999) with the proof calculus of $C R W L$, which determines the logical meaning of a FLP program, but not its execution. The operational procedure in $C R W L$ is given by a narrowing-based goal solving calculus, which is proved to be sound and complete with respect to the proof calculus. Our idea with $C R W L F$ is to follow a similar way: with the proof calculus as a guide, develop a narrowing-based operational calculus able to compute failures (even in presence of variables). We are currently working on this issue.

It is nevertheless interesting to comment that the operational approach to failure mentioned at the beginning of the section for the case of Prolog, can be also adopted for FLP, leading to a very easy implementation of failure: to evaluate fails $(e)$, try to compute a head normal form of $e$; if this fails, return true, otherwise return false. This is specially easy to be done in systems having a Prolog-based implementation like Curry or $\mathcal{T} \mathcal{O Y}$. We have checked that all the examples in Section 2 are executable in $\mathcal{T O Y}$ with this implementation of failure, if the function fails is only applied to ground expressions. For instance, the goal safe $(c) \bowtie T$ succeeds with answer $T=$ true, and safe $(a) \bowtie T$ succeeds with answer $T=$ false. If fails is applied to expressions with variables, this implementation is unsound. For instance, the goal safe $(X) \bowtie$ false succeeds without binding $X$, which is incorrect. The relationship between this kind of failure and $C R W L F$ is an interesting issue to investigate, but it is out of the scope of this paper.

The most remarkable technical insight in $C R W L F$ has been to replace the statements $e \rightarrow t$ of $C R W L$ (representing a single reduction of $e$ to an approximated value $t$ ) by $e \triangleleft \mathcal{C}$ (representing a whole, somehow complete, set $\mathcal{C}$ of approximations

to $e$ ). With the aid of $\triangleleft$ we have been able to cover all the derivations in $C R W L$, as 
well as to prove failure of reduction and, as auxiliary notions, failure of joinability and divergence, the two other kinds of statements that $C R W L$ was able to prove.

The idea of collecting into an $S A S$ values coming from different reductions for a given expression $e$ presents some similarities with abstract interpretation which, within the FLP field, has been used in Bert and Echahed (1995) for detecting unsatisfiability of equations $e=e^{\prime}$ (something similar to failure of our $e \bowtie e^{\prime}$ ). We can mention some differences between our work and Bert and Echahed (1995):

- Programs in Bert and Echahed (1995) are much more restrictive: they must be confluent, terminating, satisfy a property of stratification on conditions, and define strict and total functions.

- In our setting, each $S A S$ for an expression $e$ consists of (down) approximations to the denotation of $e$, and the set of $S A S$ 's for $e$ determines in a precise sense (Propositions 4 and 5] the denotation of $e$. In the abstract interpretation approach one typically obtains, for an expression $e$, an abstract term representing a superset of the denotation of all the instances of $e$. But some of the rules of the $C R W L F$-calculus (like (9) or (10)) are not valid if we replace $S A S$ 's by such supersets. To be more concrete, if we adopt an abstract interpretation view of our $S A S$ 's, it would be natural to see $\perp$ as standing for the set of all constructor terms (since $\perp$ is refinable to any value), and therefore to identify an $S A S$ like $\mathcal{C}=\{\perp, z\}$ with $\mathcal{C}^{\prime}=\{\perp\}$. But from $e \triangleleft \mathcal{C}$ we can deduce $e \bowtie z$, while it is not correct to do the same from $e \triangleleft \mathcal{C}^{\prime}$. Therefore, the good properties of $C R W L F$ with respect to $C R W L$ are lost.

We see our work as a step in the research of a whole framework for dealing with failure in FLP. Some natural future steps are to develop model theoretic and operational semantics for programs making use of failure information. On the practical side, we are currently working on an implementation of failure for the FLP system $\mathcal{T O Y}$ (López and Sánchez 1999a Abengózar et al. 2002).

\section{References}

Abengózar-Carneros, M. et AL. 2002. TOY: A Multiparadigm Declarative Language. Version 2.0. Departamento de Sistemas Informáticos y Programación, Universidad Complutense de Madrid, February 2002.

Antoy, S. 1997. Optimal Non-Deterministic Functional Logic Computations. In Proceedings of the 6th International Conference on Algebraic and Logic Programming (ALP'97). Springer LNCS 1298, 16-30.

Apt, K.R. 2000. Logic programming and Prolog. Unpublished tutorial.

Apt, K.R., AND BoL, R. 1994. Logic programming and negation: A survey. Journal of Logic Programming, 19\&20,9-71.

Arenas-Sánchez, P., López-Fraguas F.J. And Rodríguez-Artalejo, M. 1999. Functional plus logic programming with built-in and symbolic constraints. In Proceedings of the First International Conference onPrinciples and Practice of Declarative Programming (PPDP'99). Springer LNCS 1702, 152-169.

Arenas-SÁnchez, P. And Rodríguez-Artalejo M. 2001. A General Framework for Lazy Functional Logic, Programming with Algebraic Polymorphic Types. Theory and Practice of Logic Programming, 1(2), 185-245. 
BERT D. AND ECHAHED R. 1995. Abstraction of conditional term rewriting systems. In Proceedings of the 1995 International Logic Programming Symposium (ILPS'95). The MIT Press, 162-176.

Clark, K.L. 1978. Negation as failure. In H. Gallaire and J. Minker (eds.): Logic and Databases. Plenum Press, 293-322.

CURRY MAILING LIST. 2000. curry@informatik.rwth-aachen.de, October 2000.

González-Moreno, J.C., Hortalá-GonzÁlez, T., López-Fraguas, F.J. And Rodríguez-Artalejo, M. 1996. A Rewriting Logic for Declarative Programming. In Proceedings of the 6th European Symposium on Programming (ESOP'96). Springer LNCS 1058, 156-172.

González-Moreno, J.C., Hortalá-GonzÁlez, T., López-Fraguas, F.J. And Rodríguez-Artalejo, M. 1999. An approach to declarative programming based on a rewriting logic. Journal of Logic Programming, 40(1), 47-87.

González-Moreno, J.C., Hortalá-González, T. and Rodríguez-Artalejo, M. 1997. A higher order rewriting logic for functional logic programming. In Proceedings of the 14th International Conference on Logic Programming (ICLP'97). The MIT Press, 153-167.

Hanus, M. 1994. The integration of functions into logic programming: From theory to practice. Journal of Logic Programming, 19\&20, 583-628.

Hanus, M., Ed. 1999. Curry: An integrated functional logic language, Version 0.7.1. Available at http://www.informatik. uni-kiel.de/ mh/curry/report.html, June 2000.

J̈̈GER, G. AND STÄRK, R.F. 1998. A proof-theoretic framework for logic programming. In S.R. Buss (ed.), Handbook of Proof Theory. Elsevier, 639-682.

López-Fraguas, F.J. ANd SÁnChez-Hernández, J. 1999a. TOYY: A multiparadigm declarative system. In Proceedings of the 10th International Conference on Rewriting Techniques and Applications (RTA'99). Springer LNCS 1631, 244-247.

López-Fraguas, F.J. And SÁnchez-Hernández, J. 1999b. Disequalities may help to narrow. In Proceedings of the Joint Conference on Declarative Programming (APPIAGULP-PRODE'99), 89-104.

López-Fraguas, F.J. And SÁnchez-Hernández, J. 2000. Proving failure in functional logic programs. In Proceedings of the First International Conference on Computational Logic (CL'2000). Springer LNAI 1861, 179-193.

Moreno-Navarro, J.J. 1994. Default rules: An extension of constructive negation for narrowing-based languages. In Proceedings of the 12th International Conference on Logic Programming (ICLP'95). The MIT Press, 535-549.

Moreno-Navarro, J.J. 1996. Extending constructive negation for partial functions in lazy functional-logic languages. In Proceedings of the 5th International Workshop on Extensions of Logic Programming (ELP'96). Springer LNAI 1050, 213-227.

Peyton-Jones, S. And Hughes, J., Eds. 1999. Haskell 98: A Non-strict, Purely Functional Language. Available at http://www.haskell.org February 1999.

StuCkey P.J. 1991. Constructive negation for constraint logic programming. In Proceedings 6th Annual IEEE Symposium on Logic in Computer Science (LICS'91), 328-339.

StuCKey, P.J. 1995. Negation and constraint logic programming. Information and Computation, 118, 12-33. 